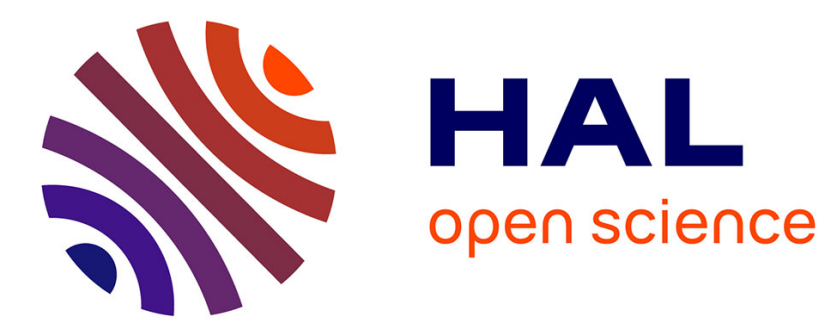

\title{
A mathematical model for a pseudo-plastic welding joint
}

\author{
Oana Iosifescu, Pongpol Juntharee, Christian Licht, Gérard Michaille
}

\section{To cite this version:}

Oana Iosifescu, Pongpol Juntharee, Christian Licht, Gérard Michaille. A mathematical model for a pseudo-plastic welding joint. Analysis and Applications, 2009, 7, pp.243-267. 10.1142/S0219530509001372 . hal-00574669

\section{HAL Id: hal-00574669 \\ https://hal.science/hal-00574669}

Submitted on 22 Oct 2012

HAL is a multi-disciplinary open access archive for the deposit and dissemination of scientific research documents, whether they are published or not. The documents may come from teaching and research institutions in France or abroad, or from public or private research centers.
L'archive ouverte pluridisciplinaire HAL, est destinée au dépôt et à la diffusion de documents scientifiques de niveau recherche, publiés ou non, émanant des établissements d'enseignement et de recherche français ou étrangers, des laboratoires publics ou privés. 


\title{
A mathematical model for a pseudo-plastic welding joint
}

\author{
Oana Iosifescu*, Pongpol Juntharee*†, Christian Licht ${ }^{\dagger}$ \\ and Gérard Michaille*
}

January 12, 2009

\begin{abstract}
An elementary situation in welding involves the perfect assembly of two adherents and a strong adhesive occupying a thin layer. The bulk energy density of the hyperelastic adherents grows superlinearly while the one of the pseudo-plastic adhesive grows linearly with a stiffness of the order of its thickness $\varepsilon$. We propose a simplified but accurate model by studying the asymptotic behavior, when $\varepsilon$ goes to zero, through variational convergence methods: at the limit, the intermediate layer is replaced by a pseudoplastic interface which allows cracks to appear.
\end{abstract}

\section{Contents}

1 Introduction

2 Description of the model 2

3 The main convergence result $\quad 8$

4 The case when $f$ is not quasiconvex and $g$ is not convex 12

5 A modeling of a welding assembly 19

6 A variational regularization of the limit functional 21

7 References $\quad 26$

*ACSIOM and AVA, UMR-CNRS 5149, Université Montpellier 2 et Université de Nîmes, Case courier 051, Place Eugène Bataillon, 34095 Montpellier Cedex 5, iosifescu@math.univmontp2.fr, micha@math.univ-montp2.fr

${ }^{\dagger}$ LMGC, UMR-CNRS 5508, Université Montpellier II, Case courier 048, Place Eugène Bataillon, 34095 Montpellier Cedex 5, Juntharee@lmgc.univ-montp2.fr, licht@lmgc.univmontp2.fr 


\section{Introduction}

Motivated by the mathematical modeling of a problem of welding, we revisit previous studies ([1], [8]) devoted to the asymptotic behavior of a structure made of two adherents connected by a thin and strong adhesive layer. In [8] the adherents and the adhesive were modeled as hyperelastic by bulk energy densities with the same growth exponent $p$ laying in $(1,+\infty)$, the stiffness of the adhesive being of the order of the inverse of its thickness. Here, our first attempt to account for some fracture phenomena in soldered joint is to model the adhesive as pseudo-plastic, that is to say its behavior is described by a bulk energy density with linear growth. Hence, from the mathematical point of view, two difficulties appear: the growths of the the bulk energy in the adhesives and the adherent are different and the linear growth in the adherent will imply to work in spaces of displacement fields with free discontinuities

The paper is organized as follows. In Section 2, we describe a model problem with a simplified geometry directly connected to the study [8] where we assume that the bulk energy density of the adherents is quasiconvex and the one of the adhesive is convex. In Section 3, a variational convergence result, when the thickness of the adhesive layer goes to zero, justifies our proposal of simplified but accurate enough model. The adhesive layer is replaced by a material pseudoplastic surface. The case when $f$ is not quasiconvex and $g$ is not convex is considered in Section 4. In Section 5, we use the previous results to model a more realistic situation of welding. Eventually, in the spirit of [7], [17], we consider a variational regularization of the limit functional involved by our model in Section 6.

\section{Description of the model}

We make no difference between $\mathbf{R}^{3}$ and the three dimensional euclidean physical space whose orthogonal basis is denoted by $\left(e_{1}, e_{2}, e_{3}\right)$, Greek coordinate indexes will run in $\{1,2\}$ and Latin ones in $\{1,2,3\}$. For all $\zeta=\left(\zeta_{1}, \zeta_{2}, \zeta_{3}\right)$ of $\mathbf{R}^{3}$, $\hat{\zeta}$ stands for $\left(\zeta_{1}, \zeta_{2}\right)$. Let $S$ be a domain of $\mathbf{R}^{2}$ with a Lipschitz-continuous boundary $\partial S$ and $r$ a positive number. The cylindrical domain $\Omega:=S \times(-r, r)$ is the reference configuration of a structure made of two adherents and an adhesive which respectively occupies $\Omega_{\varepsilon}^{ \pm}:=\left\{x \in \Omega: \pm x_{3}>\varepsilon / 2\right\}$ and $B_{\varepsilon}:=\{x \in \Omega$ : $\left.\left|x_{3}\right|<\varepsilon / 2\right\}$. We set $\Omega_{\varepsilon}:=\Omega_{\varepsilon}^{+} \cup \Omega_{\varepsilon}^{-}$. The structure is clamped on a part $\Gamma_{0}$ of the boundary $\Gamma$ of $\Omega$ with a positive $\mathcal{H}^{2}$-measure and we assume that there exists $\varepsilon_{0}>0$ such that $\operatorname{dist}\left(\bar{\Gamma}_{0}, \bar{B}_{\varepsilon_{0}}\right)>0$. The structure is subjected to body forces of density $\Phi$ and to surface forces of density $\varphi$ on the complementary part $\Gamma_{\varphi}$ of $\Gamma_{0}$. We assume that the supports of $\varphi$ and $\Phi$ lay outside of $\bar{B}_{\varepsilon_{0}}$. Obviously, one can consider other type of boundary conditions (e.g. a combination of some components of the stress vector and of the displacement). At last, adhesive and adherents are assumed to be perfectly stuck together. In section 5 we will consider a more realistic structure (see figure 1 for the two geometrical strucures). 
The stiffness of the material occupying the thin layer $B_{\varepsilon}$ is assumed to be of order $1 / \varepsilon$ so that the strain in $B_{\varepsilon}$ is expected to be small and we will use the framework of small perturbations to model the behavior of the adhesive. To account for possible fracture phenomena inside $B_{\varepsilon}$, we consider the adhesive as pseudo-plastic. Hence the behavior of the adhesive is described by a bulk energy density like $1 / \varepsilon g(e(u))$ where $g$ is a convex function with linear growth of the linearized strain $e(u)$ i.e., the symmetric part $(\nabla u)_{s}$ of the gradient displacement $\nabla u$. By contrast, the deformations in the adherents may be large and they are modeled as hyperelastic with a continuous quasiconvex bulk energy density $f$, function of the gradient displacement. More precisely, we assume that there exists $p>1$, and two positive constants $\alpha, \beta$ such that

$$
\begin{aligned}
& f, g: \mathbf{M}^{3 \times 3} \rightarrow \mathbf{R} \\
& \alpha|\xi|^{p} \leq f(\xi) \leq \beta\left(1+|\xi|^{p}\right) \text { for all } \xi \in \mathbf{M}^{3 \times 3} \\
& \alpha|\xi| \leq g(\xi) \leq \beta(1+|\xi|) \text { for all } \xi \in \mathbf{M}_{s}^{3 \times 3} .
\end{aligned}
$$

Here and in the sequel $\mathbf{M}^{n \times n}$ and $\mathbf{M}_{s}^{n \times n}$ stand for the set of $n \times n$ matrices and $n \times n$ symmetric matrices with real entries respectively. It is well known that $f$ and $g$ satisfy the following locally Lipschitz conditions: there exists a positive constant $L$ such that

$$
\begin{aligned}
& \left|f(\xi)-f\left(\xi^{\prime}\right)\right| \leq L\left|\xi-\xi^{\prime}\right|\left(1+|\xi|^{p-1}+\left|\xi^{\prime}\right|^{p-1}\right) \text { for all } \xi, \xi^{\prime} \in \mathbf{M}^{3 \times 3} \\
& \left|g(\xi)-g\left(\xi^{\prime}\right)\right| \leq L\left|\xi-\xi^{\prime}\right| \text { for all } \xi, \xi^{\prime} \in \mathbf{M}_{s}^{3 \times 3}
\end{aligned}
$$

Thus, if $F_{\varepsilon}(u):=\int_{\Omega_{\varepsilon}} f(\nabla u) d x+\frac{1}{\varepsilon} \int_{B_{\varepsilon}} g\left(e(u) d x\right.$ and $L(u):=\int_{\Omega} \Phi . u d x+$ $\int_{\Gamma_{\varphi}} \varphi \cdot u d \mathcal{H}^{2}$ respectively denote the total stored energy and the work of the external loading, determining the equilibrium configurations leads to the problem

$$
\inf \left\{\int_{\Omega_{\varepsilon}} f(\nabla u) d x+\frac{1}{\varepsilon} \int_{B_{\varepsilon}} g(e(u)) d x-L(u): u \in A_{\varepsilon}\right\}
$$

with

$$
\begin{aligned}
& A_{\varepsilon}:=\left\{u \in \operatorname{LD}\left(\Omega, \mathbf{R}^{3}\right): u_{\left\lfloor\Omega_{\varepsilon}\right.} \in W_{\Gamma_{0}}^{1, p}\left(\Omega_{\varepsilon}, \mathbf{R}^{3}\right)\right\} ; \\
& W_{\Gamma_{0}}^{1, p}\left(\Omega_{\varepsilon}, \mathbf{R}^{3}\right):=\left\{u \in W^{1, p}\left(\Omega_{\varepsilon}, \mathbf{R}^{3}\right): u=0 \text { on } \Gamma_{0}\right\} ; \\
& \operatorname{LD}\left(\Omega, \mathbf{R}^{3}\right):=\left\{u \in L^{1}\left(\Omega, \mathbf{R}^{3}\right): e(u) \in L^{1}\left(\Omega, \mathbf{M}_{s}^{3 \times 3}\right\} .\right.
\end{aligned}
$$

We aim to propose a simplified but accurate model where qualitative and quantitative analysis are able to be done in an easier way than with the starting problem. For this, we will consider $\varepsilon$ as a parameter going to zero and determine the asymptotic behavior of (approximate) solutions of the previous minimization problem by identifying the $\Gamma$-limit of $F_{\varepsilon}$ extended into the fixed space $L^{1}\left(\Omega, \mathbf{R}^{3}\right)$. More precisely, we still denote by $F_{\varepsilon}$ its extension outside $A_{\varepsilon}$ given by:

$F_{\varepsilon}: L^{1}\left(\Omega, \mathbf{R}^{3}\right) \rightarrow \mathbf{R} \cup\{+\infty\}$,

$$
F_{\varepsilon}(u):=\left\{\begin{array}{l}
\int_{\Omega_{\varepsilon}} f(\nabla u) d x+\frac{1}{\varepsilon} \int_{B_{\varepsilon}} g(e(u)) d x \text { if } u \in A_{\varepsilon} \\
+\infty \text { otherwise. }
\end{array}\right.
$$


Clearly the previous minimization problem is equivalent to the following

$$
\inf \left\{F_{\varepsilon}(u)-L(u): u \in L^{1}\left(\Omega, \mathbf{R}^{3}\right)\right\} .
$$

We will use the classical spaces

$$
\begin{aligned}
& W_{\Gamma_{0}}^{1, p}\left(\Omega, \mathbf{R}^{3}\right):=\left\{u \in W^{1, p}\left(\Omega, \mathbf{R}^{3}\right): u=0 \text { on } \Gamma_{0}\right\} ; \\
& \operatorname{BD}\left(\Omega, \mathbf{R}^{3}\right):=\left\{v \in L^{1}\left(\Omega, \mathbf{R}^{3}\right): e(v) \in \mathcal{M}\left(\Omega, \mathbf{M}_{s}^{3 \times 3}\right\} ;\right. \\
& \operatorname{BD}\left(S, \mathbf{R}^{2}\right):=\left\{v \in L^{1}\left(S, \mathbf{R}^{2}\right): e(v) \in \mathcal{M}\left(S, \mathbf{M}_{s}^{2 \times 2}\right\}\right.
\end{aligned}
$$

and the set of "horizontal rigid motions" on $S$, i.e.

$$
\begin{aligned}
\widehat{\mathcal{R}_{H}} & :=\left\{v \in \operatorname{BD}\left(S, \mathbf{R}^{2}\right): e_{\alpha \beta}(v)=0\right\} \\
& =\left\{v: v(x)=\left(a_{1}-b x_{2}, a_{2}+b x_{1}\right),\left(a_{1}, a_{2}\right) \in \mathbf{R}^{2}, b \in \mathbf{R}\right\} .
\end{aligned}
$$

For reasons clarified in Lemma 2 below, we define the limit admissible set $A_{0}$ by

$$
A_{0}:=\left\{u \in W_{\Gamma_{0}}^{1, p}\left(\Omega, \mathbf{R}^{3}\right): \gamma_{S}(\hat{u}) \in \operatorname{BD}\left(S, \mathbf{R}^{2}\right)\right\}
$$

and its subspace $A_{0}^{1}$ made of smooth elements

$$
A_{0}^{1}:=\left\{u \in A_{0}: \gamma_{S}(u) \in \mathcal{C}^{1}\left(\bar{S}, \mathbf{R}^{3}\right)\right\} .
$$

For simplicity of notation $\gamma_{S}$ will denote indifferently the trace operator from $W^{1, p}\left(\Omega, \mathbf{R}^{i}\right)$ into $W^{1-\frac{1}{p}, p}\left(S, \mathbf{R}^{i}\right)$ for $i \in\{1,2,3\}$.

For all $\xi \in \mathbf{M}_{s}^{3 \times 3}$, define $\hat{\xi} \in \mathbf{M}_{s}^{2 \times 2}$ by $(\hat{\xi})_{\alpha \beta}=\xi_{\alpha \beta}$ and consider the function $g_{0}: \mathbf{M}_{s}^{2 \times 2} \rightarrow \mathbf{R}$ defined by

$$
g_{0}(\zeta):=\min \left\{g(\xi): \xi \in \mathbf{M}_{s}^{3 \times 3}, \hat{\xi}=\zeta\right\} .
$$

It is easily seen that $g_{0}$ is a convex function on $\mathbf{M}_{s}^{2 \times 2}$ and it will be sometimes convenient to express $g_{0}$ as stated in the next lemma:

Lemma 1. For all $3 \times 2$-matrix $\xi$, $\inf _{\lambda \in \mathbf{R}^{3}} g\left((\xi \mid \lambda)_{s}\right)=g_{0}\left(\left(\xi_{\alpha \beta}\right)_{s}\right)$.

Proof. The conclusion is a straightforward consequence of the calculation

$$
\begin{aligned}
\min _{\lambda \in \mathbf{R}^{3}} g\left((\xi \mid \lambda)_{s}\right) & =\min _{\lambda \in \mathbf{R}^{3}} g\left(\left(\begin{array}{ccc}
\xi_{11} & \frac{\xi_{12}+\xi_{21}}{2} & \frac{\lambda_{1}+\xi_{31}}{2} \\
\frac{\xi_{12}+\xi_{21}}{2} & \xi_{22} & \frac{\lambda_{2}+\xi_{32}}{2} \\
\frac{\lambda_{1}+\xi_{31}}{2} & \frac{\lambda_{2}+\xi_{32}}{2} & \lambda_{3}
\end{array}\right)\right) \\
& =\min _{\lambda \in \mathbf{R}^{3}} g\left(\left(\begin{array}{ccc}
\xi_{11} & \frac{\xi_{12}+\xi_{21}}{2} & \lambda_{1} \\
\frac{\xi_{12}+\xi_{21}}{2} & \xi_{22} & \lambda_{2} \\
\lambda_{1} & \lambda_{2} & \lambda_{3}
\end{array}\right)\right)
\end{aligned}
$$

and the definition of $g_{0}$. 
In Section 3, we establish the $\Gamma$-convergence of the sequence $\left(F_{\varepsilon}\right)_{\varepsilon>0}$ to the functional defined by:

$$
\begin{gathered}
F_{0}: L^{1}\left(\Omega, \mathbf{R}^{3}\right) \rightarrow \mathbf{R} \cup\{+\infty\} \\
F_{0}(u):=\left\{\begin{array}{l}
\int_{\Omega} f(\nabla u) d x+\int_{S}\left(g _ { 0 } \left(e\left(\gamma_{s}(\hat{u})\right) \text { if } u \in A_{0}\right.\right. \\
+\infty \text { otherwise }
\end{array}\right.
\end{gathered}
$$

when $L^{1}\left(\Omega, \mathbf{R}^{3}\right)$ is equipped with its strong topology. For the defintion of the scalar measure $g_{0}(e(v)), v \in \mathrm{BD}\left(S, \mathbf{R}^{2}\right)$, we refer the reader to [12], [17], [6]. We recall that the integral over $S$ of the measure $g_{0}(e(v))$ is given by

$$
\int_{S} g_{0}(e(v))=\int_{S} g_{0}\left(e_{a}(v)\right) d \hat{x}+\int_{S} g_{0}^{\infty}\left(\frac{e_{s}(v)}{\left|e_{s}(v)\right|}\right)\left|e_{s}(v)\right|
$$

where $e(v)=e_{a}(v) d \hat{x}+e_{s}(v)$ is the Lebesgue decomposition of $e(v),\left|e_{s}(v)\right|$ denotes the total variation of the singular measure $e_{s}(v), \frac{e_{s}(v)}{\left|e_{s}(v)\right|}$ its Radon-Nikodym derivative, and $\xi \mapsto g_{0}^{\infty}(\xi):=\lim _{t \rightarrow+\infty} g_{0}(t \xi) / t$ is the recession function of $g_{0}$. In [3], Ambrosio, Coscia and Dal Maso proved that the singular measure $e_{s}(\hat{v})$ has the following structure: there exists a rectifiable set $S_{v} \subset S$ with normal $\nu_{v}$ and traces $v^{ \pm}$on both sides of $S_{v}$ such that

$$
e_{s}(v)=\frac{1}{2}\left(\left(v^{+}-v^{-}\right) \otimes \nu_{v}+\nu_{v} \otimes\left(v^{+}-v^{-}\right)\right) \mathcal{H}^{1}\left\lfloor S_{v}+C v,\right.
$$

with $C v$ singular with respect to the Lebesgue measure and vanishing on Borel sets of $\sigma$-finite $\mathcal{H}^{1}$-measure. We will also denote by $[v] \otimes_{s} \nu_{v}$ the symetrical tensor product $\left(v^{+}-v^{-}\right) \otimes \nu_{v}+\nu_{v} \otimes\left(v^{+}-v^{-}\right)$. The set $S_{v}$ will represent the macroscopic cracks whereas the support of $C_{v}$ deals with the diffuse defects or fractal cracks in $S$ towards the layer shrinks.

We start by establishing a compactness result which justifies the introduction of the limit set $A_{0}$ of admissible functions. As usual the arrows $\rightarrow$ and $\rightarrow$ will denote strong and weak convergences respectively.

Lemma 2 (Compactness lemma). Let $\left(u_{\varepsilon}\right)_{\varepsilon>0}$ be a sequence in $L^{1}\left(\Omega, \mathbf{R}^{3}\right)$ such that $\sup _{\varepsilon>0} F_{\varepsilon}\left(u_{\varepsilon}\right)<+\infty$. Then, there exists $u \in L^{1}\left(\Omega, \mathbf{R}^{3}\right)$ and a subsequence not relabelled such that

$$
\begin{aligned}
& \text { i) } u_{\varepsilon} \rightarrow u \text { in } B D\left(\Omega, \mathbf{R}^{3}\right) \text { and } u \in W_{\Gamma_{0}}^{1, p}\left(\Omega, \mathbf{R}^{3}\right) \text {; } \\
& \text { ii) } u_{\varepsilon} \rightarrow u \text { in } W_{\Gamma_{0}}^{1, p}\left(\Omega_{\eta}, \mathbf{R}^{3}\right) \text { for every } \eta>0 ; \\
& \text { iii) } \gamma_{S}(\hat{u}) \in B D\left(S, \mathbf{R}^{2}\right) \text {; } \\
& \text { iv) } \exists r_{\varepsilon} \in \widehat{\mathcal{R}_{H}} \text { such that } \frac{1}{\varepsilon} \int_{-\frac{\varepsilon}{2}}^{\frac{\varepsilon}{2}} \hat{u}_{\varepsilon} d x_{3}+r_{\varepsilon} \rightarrow \gamma_{S}(\hat{u}) \text { in } B D\left(S, \mathbf{R}^{2}\right) \text {. }
\end{aligned}
$$


Proof. From now on, we do not relabel the various subsequences obtained in the proof, and $C$ will denote a positive constant which may vary from line to line. We divide the proof into two steps.

Step 1. We establish (i) and (ii). According to the coerciveness condition (1) and because $u_{\varepsilon}=0$ on $\Gamma_{0},\left(u_{\varepsilon}\right)_{\varepsilon>0}$ is clearly bounded in $\operatorname{LD}\left(\Omega, \mathbf{R}^{3}\right)$ so that there exist $u \in \operatorname{BD}\left(\Omega, \mathbf{R}^{3}\right)$ and a subsequence satisfying $u_{\varepsilon} \rightarrow u$ in $\operatorname{BD}\left(\Omega, \mathbf{R}^{3}\right)$ and $u_{\varepsilon} \rightarrow u$ in $L^{1}\left(\Omega, \mathbf{R}^{3}\right)$. The weak convergence of $u_{\varepsilon}$ to $u$ in $W_{\Gamma_{0}}^{1, p}\left(\Omega_{\eta}, \mathbf{R}^{3}\right)$ for every $\eta>0$ is obvious. We are going to prove that $u \in W_{\Gamma_{0}}^{1, p}\left(\Omega, \mathbf{R}^{3}\right)$.

We extend every function $w \in W_{\Gamma_{0}}^{1, p}\left(\Omega_{\varepsilon}, \mathbf{R}^{3}\right)$ by reflexion on $S \times\left( \pm r, \pm 2 r \mp \frac{\varepsilon}{2}\right)$ so that the extended function, denoted by $\tilde{w}$, belongs to $W^{1, p}\left(S \times\left( \pm \frac{\varepsilon}{2}, \pm 2 r \mp\right.\right.$ $\left.\frac{\varepsilon}{2}\right), \mathbf{R}^{3}$ ) and satisfies

$$
\int_{S \times\left( \pm \frac{\varepsilon}{2}, \pm 2 r \mp \frac{\varepsilon}{2}\right)}|\nabla \tilde{w}|^{p} d x \leq 2 \int_{\Omega_{\varepsilon}^{ \pm}}|\nabla w|^{p} d x .
$$

Set $\Omega^{ \pm}:=\Omega \cap\left[ \pm x_{3}>0\right]$ and, for every function $w \in W_{\Gamma_{0}}^{1, p}\left(\Omega_{\varepsilon}, \mathbf{R}^{3}\right)$, define its $\varepsilon$-translate $T_{\varepsilon} w$ in $W^{1, p}\left(\Omega \backslash S, \mathbf{R}^{3}\right)$ by

$$
T_{\varepsilon} w\left(\hat{x}, x_{3}\right)= \begin{cases}\tilde{w}\left(\hat{x}, x_{3}+\varepsilon / 2\right), & \text { if } x \in \Omega^{+} \\ \tilde{w}\left(\hat{x}, x_{3}-\varepsilon / 2\right), & \text { if } x \in \Omega^{-} .\end{cases}
$$

Because

$$
\begin{aligned}
\sup _{\varepsilon>0} \int_{\Omega \backslash S}\left|\nabla T_{\varepsilon} u_{\varepsilon}\right|^{p} d x & \leq \sup _{\varepsilon>0} \int_{S \times\left(\frac{\varepsilon}{2}, 2 r-\frac{\varepsilon}{2}\right) \cup S \times\left(-2 r+\frac{\varepsilon}{2},-\frac{\varepsilon}{2}\right)}\left|\nabla \tilde{u}_{\varepsilon}\right|^{p} d x \\
& \leq 2 \sup _{\varepsilon>0} \int_{\Omega_{\varepsilon}}\left|\nabla u_{\varepsilon}\right|^{p} d x<+\infty,
\end{aligned}
$$

Poincaré's inequality implies that there exist $z \in W^{1, p}\left(\Omega \backslash S, \mathbf{R}^{3}\right)$ and a subsequence of $\left(u_{\varepsilon}\right)_{\varepsilon>0}$ such that $T_{\varepsilon} u_{\varepsilon} \rightarrow z$ in $W^{1, p}\left(\Omega \backslash S, \mathbf{R}^{3}\right)$ and $u_{\varepsilon} \rightarrow z$ in $L^{p}\left(\Omega, \mathbf{R}^{3}\right)$. Actually $u=z$ (so that $u \in W^{1, p}\left(\Omega \backslash S, \mathbf{R}^{3}\right)$ ) since for all $\psi \in \mathcal{D}\left(\Omega \backslash S, \mathbf{R}^{m}\right)$,

$$
\begin{aligned}
\int_{\Omega \backslash S} u . \psi d x & =\lim _{\varepsilon \rightarrow 0} \int_{\Omega \backslash S} u_{\varepsilon} \cdot \psi\left(\hat{x}, x_{3}-\frac{\varepsilon}{2}\right) d x \\
& =\lim _{\varepsilon \rightarrow 0} \int_{\Omega \backslash S} T_{\varepsilon} u_{\varepsilon} \cdot \psi d x \\
& =\int_{\Omega \backslash S} z \cdot \psi d x .
\end{aligned}
$$

For all $w \in W^{1, p}\left(\Omega \backslash S, \mathbf{R}^{3}\right)$, we will denote the traces on $S$ of $w$ considered as a function of $W^{1, p}\left(\Omega^{ \pm}, \mathbf{R}^{3}\right)$ by $w^{ \pm}$and its jump accross $S$ by $[w]:=w^{+}-w^{-}$. Take $\left.\theta \in \mathbf{C}_{c}^{\infty}(S)\right)$, Green's formula yields

$$
2 \int_{B_{\varepsilon}} e_{\alpha 3}\left(u_{\varepsilon}\right) \theta d x=\int_{S} \theta\left[T_{\varepsilon} u_{\alpha}^{\varepsilon}\right] d \hat{x}+I_{\varepsilon}
$$


where

$$
I_{\varepsilon}:=-\int_{B_{\varepsilon}} \frac{\partial u_{3}^{\varepsilon}}{\partial x_{\alpha}} \theta d x
$$

The left hand side term of (5) tends to 0 by coercivity condition (2), and from

$$
\begin{aligned}
u_{3}^{\varepsilon}\left(\hat{x}, \pm\left|x_{3}\right|\right) & =u_{3}^{\varepsilon}(\hat{x}, \pm \varepsilon / 2)+\int_{ \pm \varepsilon / 2}^{ \pm\left|x_{3}\right|} \frac{\partial u_{3}^{\varepsilon}}{\partial x_{3}}(\hat{x}, t) d t \\
& =\left(T_{\varepsilon} u_{3}^{\varepsilon}\right)^{ \pm}(\hat{x})+\int_{ \pm \varepsilon / 2}^{ \pm\left|x_{3}\right|} \frac{\partial u_{3}^{\varepsilon}}{\partial x_{3}}(\hat{x}, t) d t
\end{aligned}
$$

we deduce

$$
\begin{aligned}
\int_{B_{\varepsilon}}\left|u_{3}^{\varepsilon}\left(\hat{x}, x_{3}\right)\right| d x & \leq \varepsilon \int_{S}\left(\left|\left(T_{\varepsilon} u_{3}^{\varepsilon}\right)^{+}(\hat{x})\right|+\left|\left(T_{\varepsilon} u_{3}^{\varepsilon}\right)^{-}(\hat{x})\right|\right) d \hat{x}+\varepsilon \int_{B_{\varepsilon}}\left|\frac{\partial u_{3}^{\varepsilon}}{\partial x_{3}}\right| d x \\
& \leq C \varepsilon(1+\varepsilon)
\end{aligned}
$$

so that

$$
\left|I_{\varepsilon}\right|=\left|\int_{B_{\varepsilon}} u_{3}^{\varepsilon} \frac{\partial \theta}{\partial x_{\alpha}} d x\right| \leq C \varepsilon(1+\varepsilon),
$$

and the right hand side term of (5) tends to $\int_{S} \theta\left[u_{\alpha}\right] d \hat{x}$. Going to the limit on $\varepsilon$ in $(5)$ yields $\left[u_{\alpha}\right]=0$ a.e. on $S$. Similarly, by letting $\varepsilon \rightarrow 0$ in

$$
\int_{B_{\varepsilon}} \frac{\partial u_{3}^{\varepsilon}}{\partial x_{3}} \theta d x=\int_{S} \theta\left[T_{\varepsilon} u_{3}^{\varepsilon}\right] d \hat{x}
$$

and by using coercivity condition (2), we obtain $\left[u_{3}\right]=0$ a.e. on $S$.

Step 2. We establish (iii) and (iv). Coercivity condition (2) implies

$$
\sup _{\varepsilon>0} \frac{1}{\varepsilon} \int_{B_{\varepsilon}}\left|\widehat{e\left(u_{\varepsilon}\right)} d x\right|<+\infty
$$

so that there exists a subsequence of $v_{\varepsilon}:=\frac{1}{\varepsilon} \int_{-\frac{\varepsilon}{2}}^{\frac{\varepsilon}{2}} \hat{u}_{\varepsilon} d x_{3}, r_{\varepsilon} \in \widehat{\mathcal{R}_{H}}$ and $v \in$ $\mathrm{BD}\left(S, \mathbf{R}^{2}\right)$ such that $v_{\varepsilon}+r_{\varepsilon} \rightarrow v$ in $\operatorname{BD}\left(S, \mathbf{R}^{2}\right)^{2}$. It remains to prove that $v=\gamma_{S}(\hat{u})$. For arbitrary $\theta \in \mathcal{C}_{c}^{\infty}(S)$ and $x_{3} \in\left(-\frac{\varepsilon}{2}, \frac{\varepsilon}{2}\right)$, one has

$$
\left.u_{\alpha}^{\varepsilon}\left(\hat{x}, \pm\left|x_{3}\right|\right) \theta(\hat{x})=\left(T_{\varepsilon} u_{\alpha}^{\varepsilon}\right)^{ \pm}(\hat{x})\right) \theta(\hat{x})+\int_{ \pm \varepsilon / 2}^{ \pm\left|x_{3}\right|} \frac{\partial u_{\alpha}^{\varepsilon}}{\partial x_{3}}(\hat{x}, t) \theta(\hat{x}) d t
$$

so that

$$
\left.\frac{1}{\varepsilon} \int_{-\frac{\varepsilon}{2}}^{\frac{\varepsilon}{2}} \int_{S} u_{\alpha}^{\varepsilon}\left(\hat{x}, x_{3}\right) \theta(\hat{x}) d x=\frac{1}{2} \int_{S}\left(T_{\varepsilon} u_{\alpha}^{\varepsilon}\right)^{+}(\hat{x})+\left(T_{\varepsilon} u_{\alpha}^{\varepsilon}\right)^{+}(\hat{x})\right) \theta(\hat{x}) d \hat{x}+J_{\varepsilon}
$$

where

$J_{\varepsilon}:=\frac{1}{\varepsilon} \int_{-\frac{\varepsilon}{2}}^{0} \int_{S} \int_{-\varepsilon / 2}^{-\left|x_{3}\right|} \frac{\partial u_{\alpha}^{\varepsilon}}{\partial x_{3}}(\hat{x}, t) \theta(\hat{x}) d t d x+\frac{1}{\varepsilon} \int_{0}^{\frac{\varepsilon}{2}} \int_{S} \int_{\varepsilon / 2}^{\left|x_{3}\right|} \frac{\partial u_{\alpha}^{\varepsilon}}{\partial x_{3}}(\hat{x}, t) \theta(\hat{x}) d t d x$. 
We claim that $\lim _{\varepsilon \rightarrow 0} J_{\varepsilon}=0$. Indeed

$$
\begin{aligned}
\int_{S} \int_{\varepsilon / 2}^{x_{3}} \frac{\partial u_{\alpha}^{\varepsilon}}{\partial x_{3}} \theta(\hat{x}) d t d \hat{x} & =\int_{S} \int_{\varepsilon / 2}^{x_{3}}\left(2 e_{3 \alpha}\left(u_{\varepsilon}\right)-\frac{\partial u_{3}^{\varepsilon}}{\partial x_{\alpha}}\right) \theta d t d \hat{x} \\
& =\int_{S} \int_{\varepsilon / 2}^{x_{3}}\left(2 e_{3 \alpha}\left(u_{\varepsilon}\right) \theta+u_{3}^{\varepsilon} \frac{\partial \theta}{\partial x_{\alpha}}\right) d t d \hat{x}
\end{aligned}
$$

thus

$$
\left.\left|J_{\varepsilon}\right| \leq C \int_{B_{\varepsilon}}\left(\left|e_{3 \alpha}\left(u_{\varepsilon}\right)\right|+\mid u_{3}^{\varepsilon}\right) \mid\right) d x
$$

and the claim follows from (6) and coercivity condition (2). Letting $\varepsilon \rightarrow 0$ in (7), we obtain

$$
\int_{S} v_{\alpha} \theta d \hat{x}=\int_{S} \gamma_{S}\left(u_{\alpha}\right) \theta d \hat{x}
$$

thus $v_{\alpha}=\gamma_{S}\left(u_{\alpha}\right)$ a.e. in $S$ since $\theta$ is arbitrary.

\section{The main convergence result}

The main result of this section is the following theorem.

Theorem 1. The sequence $\left(F_{\varepsilon}\right)_{\varepsilon>0} \Gamma$-converges to the functional $F_{0}$.

The proof consists in establishing Proposition 1 and Proposition 2 below, corresponding to the lower bound and the upper bound in the definition of the $\Gamma_{\tau_{s}}$-convergence.

Proposition 1 (Lower bound). For all $u$ and all sequence $\left(u_{\varepsilon}\right)_{\varepsilon>0}$ in $L^{1}\left(\Omega, \mathbf{R}^{3}\right)$ such that $u_{\varepsilon} \stackrel{\tau_{s}}{\rightarrow} u$, the following inequality holds:

$$
F_{0}(u) \leq \liminf _{\varepsilon \rightarrow 0} F_{\varepsilon}\left(u_{\varepsilon}\right) .
$$

Proof. Clearly one may assume that $\liminf _{\varepsilon \rightarrow 0} F_{\varepsilon}\left(u_{\varepsilon}\right)<+\infty$ so that from Lemma $2, u$ belongs to $A_{0}$, and $e\left(\frac{1}{\varepsilon} \int_{-\frac{\varepsilon}{2}}^{\frac{\varepsilon}{2}} \hat{u}_{\varepsilon} d x_{3}\right)=\frac{1}{\varepsilon} \int_{-\frac{\varepsilon}{2}}^{\frac{\varepsilon}{2}} \widehat{e\left(u_{\varepsilon}\right)} d x_{3} \rightarrow e\left(\gamma_{S}(\hat{u})\right)$ in $\mathcal{M}\left(S, \mathbf{M}_{s}^{2 \times 2}\right)$. Thus from Jensen's inequality

$$
\begin{aligned}
\liminf _{\varepsilon \rightarrow 0} F_{\varepsilon}\left(u_{\varepsilon}\right) & =\liminf _{\varepsilon \rightarrow 0}\left(\int_{\Omega_{\varepsilon}} f\left(\nabla u_{\varepsilon}\right) d x+\frac{1}{\varepsilon} \int_{B_{\varepsilon}} g\left(e\left(u_{\varepsilon}\right)\right) d x\right. \\
& \geq \liminf _{\varepsilon \rightarrow 0} \int_{\Omega_{\varepsilon}} f\left(\nabla u_{\varepsilon}\right) d x+\liminf _{\varepsilon \rightarrow 0} \frac{1}{\varepsilon} \int_{B_{\varepsilon}} g\left(e\left(u_{\varepsilon}\right)\right) d x \\
& \geq \liminf _{\varepsilon \rightarrow 0} \int_{\Omega_{\varepsilon}} f\left(\nabla u_{\varepsilon}\right) d x+\liminf _{\varepsilon \rightarrow 0} \frac{1}{\varepsilon} \int_{B_{\varepsilon}} g_{0}\left(\widehat{e\left(u_{\varepsilon}\right)}\right) d x \\
& \geq \int_{\Omega} f(\nabla u) d x+\int_{S} g_{0}\left(e\left(\gamma_{S}(\hat{u})\right) .\right.
\end{aligned}
$$

In the last inequality, we used the weak lower semicontinuity of the second integral functional with respect to the weak convergence of measures. For the 
convergence of the first integral, we proceeded as follows: take $\eta>\varepsilon$, write $\int_{\Omega_{\varepsilon}} f\left(\nabla u_{\varepsilon}\right) d x \geq \int_{\Omega_{\eta}} f\left(\nabla u_{\varepsilon}\right) d x$, and apply the lower semicontinuity of the integral functional $u \mapsto \int_{\Omega_{\eta}} f(\nabla u) d x$ for the weak convergence in $W^{1, p}\left(\Omega_{\eta}, \mathbf{R}^{3}\right)$. Then let $\eta \rightarrow 0$.

For proving the upper bound, we need to establish the following relaxation result.

Lemma 3 (Relaxation). The functional $F_{0}$ is the l.s.c. regularization for the strong topology of the functional defined on $L^{1}\left(\Omega, \mathbf{R}^{3}\right)$ by

$$
\tilde{F}_{0}(u):=\left\{\begin{array}{l}
\int_{\Omega} f(\nabla u) d x+\int_{S} g_{0}\left(e\left(\gamma_{S}(\hat{u})\right) d \hat{x} \text { if } u \in A_{0}^{1},\right. \\
+\infty \text { otherwise. }
\end{array}\right.
$$

Proof. Step 1. By using standard lower semicontinuous arguments, it is easily seen that for every sequence $\left(u_{n}\right)_{n \in \mathbf{N}}$ strongly converging to $u$ in $L^{1}\left(\Omega, \mathbf{R}^{3}\right)$, one has

$$
F_{0}(u) \leq \liminf _{n \rightarrow+\infty} \tilde{F}_{0}\left(u_{n}\right) .
$$

Step 2. We assume $u \in A_{0}$ and we construct a sequence $\left(u_{\delta}\right)_{\delta>0}$ in $A_{0}^{1}$ such that

$$
\left\{\begin{array}{l}
u_{\delta} \rightarrow u \text { strongly in } W^{1, p}\left(\Omega, \mathbf{R}^{3}\right) ; \\
\gamma_{S}\left(\hat{u}_{\delta}\right)=v_{\delta} \rightarrow v:=\gamma_{S}(\hat{u}) \text { strongly in } L^{1}\left(S, \mathbf{R}^{2}\right) \\
\lim _{\delta \rightarrow 0} \int_{\Omega} f\left(\nabla u_{\delta}\right) d x=\int_{\Omega} f(\nabla u) d x \\
\lim _{\delta \rightarrow 0} \int_{S} g_{0}\left(e\left(v_{\delta}\right)\right) d \hat{x}=\int_{S} g_{0}(e(v)) .
\end{array}\right.
$$

Consider the open cylinder $\tilde{\Omega}:=\tilde{S} \times(-r, r)$ containing $\Omega$, where $\tilde{S}$ is an open set of $\mathbf{R}^{2}$ strictly containing $S$ and extend $u$ into a function $\tilde{u}$ in $W^{1, p}\left(\tilde{\Omega}, \mathbf{R}^{3}\right)$. We also extend $v$ into a function $\tilde{v}$ in $\operatorname{BV}\left(\tilde{S}, \mathbf{R}^{3}\right)$. More precisely, let $P_{3}$ denote the extension operators

$$
P_{3}: W^{1, p}\left(\Omega, \mathbf{R}^{3}\right) \rightarrow W^{1, p}\left(\tilde{\Omega}, \mathbf{R}^{3}\right),
$$

and $\gamma_{\tilde{S}}$ the trace operator associated with the Sobolev space $W^{1, p}\left(\tilde{\Omega}, \mathbf{R}^{3}\right)$. We define $\tilde{v}$ in $\operatorname{BV}\left(\tilde{S}, \mathbf{R}^{3}\right)$ by $\tilde{v}:=\gamma_{\tilde{S}} \circ P_{3}(\hat{u})$, i.e., $\tilde{v}=\gamma_{\tilde{S}}(\hat{\tilde{u}})$.

For all $\delta>0$, consider the open ball $B_{\eta(\delta)}(0)$ of $\mathbf{R}^{2}$ centered at 0 with a radius $\eta(\delta)>0$ small enough so that $S+B_{\eta(\delta}(0) \subset \tilde{S}$. Let $\phi$ in $\mathcal{C}_{c}^{\infty}(\tilde{S})$ with $\phi=1$ on a neighborhood $S+B_{\eta(\delta}(0)$ included in $\tilde{S}$ and $\rho_{\eta(\delta)}$ a standard mollifier with support $\bar{B}_{\eta(\delta)}(0)$. Set $v_{\delta}:=\rho_{\eta(\delta)} *(\phi \tilde{v}), u_{\delta}:=\rho_{\eta(\delta)} *(\phi \tilde{u})$ and choose $\eta(\delta)$ small enough so that:

$$
\begin{aligned}
& \left\|v_{\delta}-v\right\|_{L^{1}\left(S, \mathbf{R}^{2}\right)}<\delta ; \\
& \left|\int_{\mathbf{R}^{2}} g_{0}\left(\rho_{\eta(\delta)} *(\phi e(\tilde{v}))\right)-\int_{\mathbf{R}^{2}} g_{0}(\phi e(\tilde{v}))\right|<\delta ; \\
& \left\|u_{\delta}-u\right\|_{W^{1, p}\left(\Omega, \mathbf{R}^{3}\right)}<\delta .
\end{aligned}
$$


Estimates (9) and (11) are standard (note that the mollification of $\tilde{u}$ takes place only on the $\hat{x}$ argument). Estimate (10) is a straightforward consequence of the narrow convergence of the measure $g_{0}\left(\rho_{\eta(\delta)} *(\phi e(\tilde{v}))\right)$ to the measure $g_{0}(\phi e(\tilde{v}))$ in $\mathbf{M}^{+}\left(\mathbf{R}^{2}\right)$ (see for instance Lemma 5.2 and Remark 5.1 in [17], or, if $g_{0}$ is positively homogeneous of degree 1, use Reshetnyak's continuity theorem, Theorem 2.39 in [4]).

Clearly $v_{\delta} \in \mathcal{C}^{\infty}\left(\bar{S}, \mathbf{R}^{2}\right)$ and, from $(9), v_{\delta} \rightarrow v$ in $L^{1}\left(S, \mathbf{R}^{2}\right)$. Moreover, from (10), and noticing that $\phi=1$ on $S+B_{\eta(\delta)}(0)$,

$$
\left|\int_{S} g_{0}\left(e\left(v_{\delta}\right)\right)-\int_{S} g_{0}(e(v))\right| \leq \mid \int_{\mathbf{R}^{2}} g_{0}\left(\rho_{\eta(\delta)} *(\phi e(\tilde{v}))-\int_{\mathbf{R}^{2}} g_{0}(\phi e(\tilde{v})) \mid<\delta,\right.
$$

so that

$$
\lim _{\delta \rightarrow 0} \int_{S} g_{0}\left(e\left(v_{\delta}\right)\right)=\int_{S} g_{0}(e(v)) .
$$

From (11), $u_{\delta} \rightarrow u$ in $W^{1, p}\left(\Omega, \mathbf{R}^{3}\right)$ and, from (3),

$$
\lim _{\delta \rightarrow 0} \int_{\Omega} f\left(\nabla u_{\delta}\right) d x=\int_{\Omega} f(\nabla u) d x .
$$

According to the definition of $v_{\delta}, u_{\delta}$, and from the fact that $\tilde{v}=\gamma_{\tilde{S}}(\hat{\tilde{u}})$, one has $\gamma_{S}\left(\hat{u}_{\delta}\right)=v_{\delta}$.

The sequence $\left(u_{\delta}\right)_{\delta>0}$ fulfills all the conditions of the set $A_{0}^{1}$ except the boundary condition. By using De Giorgi's slicing method in a neighborhood of $\Gamma_{0}$ (see for instance Theorem 11.2.1 in [6]), one can modify $u_{\delta}$ into a new function $\tilde{u}_{\delta} \in A_{0}^{1}$ which has the same trace as its weak limit $u$ on $\partial \Omega$, and satisfies

$$
\limsup _{\delta \rightarrow 0} \int_{\Omega} f\left(\nabla \tilde{u}_{\delta}\right) d x \leq \int_{\Omega} f\left(\nabla u_{\delta}\right) d x
$$

thus, finally

$$
\lim _{\delta \rightarrow 0} \int_{\Omega} f\left(\nabla \tilde{u}_{\delta}\right) d x=\int_{\Omega} f(\nabla u) d x .
$$

Note that $u_{\delta}$ is not affected on a neighborhood of $S$ by this modification because $\operatorname{dist}\left(\bar{\Gamma}_{0}, \overline{\partial B_{\varepsilon} \cap \Gamma}\right)>0$. Thus $\tilde{u}_{\delta}$ that we denote now by $u_{\delta}$ is the expected sequence.

Proposition 2 (Upper bound). The following inequality holds in $L^{1}\left(\Omega, \mathbf{R}^{3}\right)$

$$
\left(\Gamma-\lim \sup F_{\varepsilon}\right) \leq F_{0} .
$$

Proof. Step 1. We establish $\Gamma-\lim \sup F_{\varepsilon} \leq \tilde{F}_{0}$.

Take $u \in A_{0}^{1}$. In what follows we set $v:=\gamma_{S}(\hat{u})$. For every fixed $\xi$ in $\mathcal{D}\left(S, \mathbf{R}^{3}\right)$, consider the function $v_{\varepsilon}$ in $W^{1, p}\left(B, \mathbf{R}^{3}\right), B=S \times\left(-\frac{1}{2}, \frac{1}{2}\right)$ defined by

$$
\left\{\begin{array}{l}
v_{\varepsilon}^{\alpha}\left(\hat{x}, x_{3}\right):=v^{\alpha}(\hat{x})+\varepsilon x_{3} \xi^{\alpha}(\hat{x}) \\
v_{\varepsilon}^{3}\left(\hat{x}, x_{3}\right):=\varepsilon u^{3}(\hat{x}, 0)+\varepsilon^{2} x_{3} \xi^{3}(\hat{x})
\end{array}\right.
$$


and set

$$
u_{\varepsilon}\left(\hat{x}, x_{3}\right):=\left\{\begin{array}{l}
u\left(\hat{x}, x_{3}-\frac{\varepsilon}{2}\right)+\frac{\varepsilon}{2} \xi(\hat{x}) \text { in } \Omega_{\varepsilon}^{+} \\
v_{\varepsilon}\left(\hat{x}, \frac{x_{3}}{\varepsilon}\right) \text { in } B_{\varepsilon} \\
u\left(\hat{x}, x_{3}+\frac{\varepsilon}{2}\right)-\frac{\varepsilon}{2} \xi(\hat{x}) \text { in } \Omega_{\varepsilon}^{-} .
\end{array}\right.
$$

Clearly $u_{\varepsilon} \in A_{\varepsilon}$ except the boundary condition and $u_{\varepsilon} \rightarrow u$ in $L^{1}\left(\Omega, \mathbf{R}^{3}\right)$. An easy calculation and the local Lipschitz conditions (3), (4), yield

$$
\lim _{\varepsilon \rightarrow 0} \int_{\Omega_{\varepsilon}} f\left(\nabla u_{\varepsilon}\right) d x=\int_{\Omega} f(\nabla u) d x
$$

and

$$
\begin{aligned}
\lim _{\varepsilon \rightarrow 0} \frac{1}{\varepsilon} \int_{B_{\varepsilon}} g\left(e\left(u_{\varepsilon}\right)\right) d x & =\lim _{\varepsilon \rightarrow 0} \int_{B} g\left(\left(\hat{\nabla} v_{\varepsilon} \mid \frac{1}{\varepsilon} \frac{\partial v_{\varepsilon}}{\partial x_{3}}\right)_{s}\right) d x \\
& =\int_{S} g\left((\hat{\nabla} v \mid \xi)_{s}\right) d \hat{x}
\end{aligned}
$$

so that

$$
\lim _{\varepsilon \rightarrow 0} F_{\varepsilon}\left(u_{\varepsilon}\right)=\int_{\Omega} f(\nabla u) d x+\int_{S} g\left((\hat{\nabla} v \mid \xi)_{s}\right) d \hat{x} .
$$

By using again De Giorgi's slicing method in a neighborhood of $\Gamma_{0}$, one can modify $u_{\varepsilon}$ into a function still denoted by $u_{\varepsilon}$, satisfying the boundary condition on $\Gamma_{0}$ and

$$
\lim _{\varepsilon \rightarrow 0} F_{\varepsilon}\left(u_{\varepsilon}\right)=\int_{\Omega} f(\nabla u) d x+\int_{S} g\left((\hat{\nabla} v \mid \xi)_{s}\right) d \hat{x} .
$$

According to a well known interchange result between infimum and integral (see [2]) we have

$$
\int_{S} g_{0}(e(v)) d \hat{x}=\inf _{\xi \in \mathcal{D}\left(S, \mathbf{R}^{3}\right)} \int_{S} g\left((\hat{\nabla} v \mid \xi)_{s}\right) d \hat{x} .
$$

By taking the infimum over all $\xi \in \mathcal{D}\left(S, \mathbf{R}^{3}\right)$ in (13) and by using Lemma 1 we deduce

$$
\inf \left\{\limsup _{\varepsilon \rightarrow 0} F_{\varepsilon}\left(u_{\varepsilon}\right): u_{\varepsilon} \rightarrow u \text { in } L^{1}\left(\Omega, \mathbf{R}^{3}\right)\right\} \leq \tilde{F}_{0}(u),
$$

i.e. $\Gamma-\lim \sup F_{\varepsilon} \leq \tilde{F}_{0}$.

Step 2. Taking the lower semicontinuous envelope of each two functionals for the strong topology of $L^{1}\left(\Omega, \mathbf{R}^{3}\right)$, the conclusion then follows from the lower semicontinuity of $\Gamma-\lim \sup F_{\varepsilon}$ and from Lemma 3 .

According to Lemma 2 and to variational properties of the $\Gamma$-convergence, we obtain : 
Corollary 1. Let $\bar{u}_{\varepsilon}$ be a solution of $\left(\mathcal{P}_{\varepsilon}\right)$. Then there exist a subsequence of $\left(\bar{u}_{\varepsilon}\right)_{\varepsilon>0}$ and $\bar{u}$ in $W_{\Gamma_{0}}^{1, p}\left(\Omega, \mathbf{R}^{3}\right)$ such that

$$
\begin{aligned}
& \bar{u}_{\varepsilon} \rightarrow \bar{u} \text { in } B D\left(\Omega, \mathbf{R}^{3}\right) ; \\
& \bar{u}_{\varepsilon} \rightarrow \bar{u} \text { in } W_{\Gamma_{0}}^{1, p}\left(\Omega_{\eta}, \mathbf{R}^{3}\right) \text { for every } \eta>0 ; \\
& \gamma_{S}(\hat{\bar{u}}) \in B D\left(S, \mathbf{R}^{2}\right) .
\end{aligned}
$$

Moreover $\bar{u}$ is solution of the minimization problem

$$
\min \left\{F_{0}(u)-L(u): u \in L^{1}\left(\Omega, \mathbf{R}^{3}\right)\right\}
$$

and

$$
\min \left\{F_{\varepsilon}(u)-L(u): u \in L^{1}\left(\Omega, \mathbf{R}^{3}\right)\right\} \rightarrow \min \left\{F_{0}(u)-L(u): u \in L^{1}\left(\Omega, \mathbf{R}^{3}\right)\right\} .
$$

Thus, in this simplified case (see Section 5 for a realistic geometry), our proposal of model is given by the limit problem $(\mathcal{P})$ which describes the equilibrium of a structure made of two adherents perfectly stuck to a material surface. The reference configuration of the adherents are $\Omega^{ \pm}:=\Omega \cap\left[ \pm x_{3}>0\right]$ while the one of the material surface is $S$. The adherents are hyperelastic with bulk energy density $f$ and the material surface is pseudo-plastic with surface density $g_{0}$. Due to the linear growth of $g_{0}$, the displacement field solution of $(\mathcal{P})$ may present discontinuities in $S$ which may be interpreted in terms of cracks. It is worthwhile to note that this situation with strong adhesive layer is completely different from the one considered in [15] with a soft adhesive: in the asymptotic model, the soft adhesive layer is replaced by a mechanical constraint between the adherents, whereas the strong adhesive layer is replaced by a material surface perfectly stuck to adherents. Another strategy proposed in [7] leads to a similar model.

\section{The case when $f$ is not quasiconvex and $g$ is not convex}

In this section, we drop the quasiconvex and convex assumptions on the density functions $f$ and $g$ respectively. This is the case when the materials undergo reversible solid/solid phase transformations, for which the density functions present a multi-well structure (for $f$ in the large deformation setting see [10], for $g$ in the setting of small perturbations see [13]). However we assume that $f$ and $g$ satisfy the locally Lipschitz conditions (3), (4) and that $g$ is positively 1-homogeneous. In this more general situation, we would like to show that the limit energy functional is given by

$$
\begin{gathered}
F_{0}: L^{1}\left(\Omega, \mathbf{R}^{3}\right) \rightarrow \mathbf{R} \cup\{+\infty\} \\
F_{0}(u):=\left\{\begin{array}{l}
\int_{\Omega} Q f(\nabla u) d x+\int_{S} S Q g_{0}\left(e\left(\gamma_{s}(\hat{u})\right) \text { if } u \in A_{0},\right. \\
+\infty \text { otherwise }
\end{array}\right.
\end{gathered}
$$


where $Q f$ is the quasiconvex envelope of $f$ and $S Q g_{0}: \mathbf{M}_{s}^{2 \times 2} \rightarrow \mathbf{R}$ is the symmetric quasiconvex envelope of $g_{0}$ defined by

$$
S Q g_{0}(\zeta):=\inf \left\{\frac{1}{|\hat{D}|} \int_{\hat{D}} g_{0}(\zeta+e(\varphi)) d \hat{x}: \varphi \in \mathcal{C}_{0}^{\infty}\left(\hat{D}, \mathbf{R}^{2}\right)\right\}
$$

Let denote the operator $\zeta \mapsto \zeta_{s}$ from $\mathbf{M}^{2 \times 2}$ into $\mathbf{M}_{s}^{2 \times 2}$ by $\mathcal{S}_{2}$. Thus, for every $\zeta \in \mathbf{M}_{s}^{2 \times 2}, S Q g_{0}(\zeta)=Q\left(g_{0} \circ \mathcal{S}_{2}\right)(\zeta)$ where $Q\left(g_{0} \circ \mathcal{S}_{2}\right)$ is the quasiconvex envelope of $g_{0} \circ \mathcal{S}_{2}$. Note that the right hand side term does not depend on the choice of the cube $\hat{D}$ of $\mathbf{R}^{2}$ and that $S Q g_{0}$ is 1-homogeneous. With the notation of Section 2, the integral over $S$ of the measure $S Q g_{0}(e(v))$ is given by

$$
\int_{S} S Q g_{0}(e(v))=\int_{S} S Q g_{0}\left(e_{a}(v)\right) d \hat{x}+\int_{S} S Q g_{0}\left(\frac{\left(e_{s}(v)\right.}{\left|e_{s}(v)\right|}\right)\left|e_{s}(v)\right| .
$$

Like in Section 2, we prove Proposition 3 and Proposition 5 below, corresponding to the lower and the upper bound in the definition of the $\Gamma$-convergence. Unfortunately, we establish the lower bound when $u$ belongs to the subset $\tilde{A}_{0}$ of $A_{0}$ defined by

$$
\tilde{A}_{0}:=\left\{u \in W_{\Gamma_{0}}^{1, p}\left(\Omega, \mathbf{R}^{3}\right): \gamma_{S}(\hat{u}) \in \operatorname{SBD}\left(S, \mathbf{R}^{2}\right)\right\}
$$

where $\operatorname{SBD}\left(S, \mathbf{R}^{2}\right)$ denotes the set of the elements $u$ of $\operatorname{BD}\left(S, \mathbf{R}^{2}\right)$ whose the Cantor part of the strain tensor $e(u)$ is zero. Then, the main result of this section is

Theorem 2. The restriction of the $\Gamma$-limit of $F_{\varepsilon}$ to the set $\tilde{A}_{0}$ is given by

$$
F_{0}(u)=\int_{\Omega} Q f(\nabla u) d x+\int_{S} S Q g_{0}\left(e_{a}\left(\gamma_{S}(\hat{u})\right)\right) d \hat{x}+\int_{S} S Q g_{0}\left(\left[\gamma_{s}(\hat{u})\right] \otimes_{s} \nu_{\gamma_{S}}(\hat{u})\right) d \mathcal{H}^{1} .
$$

Remark 1. If we assume that an approximate minimizer of $\left(\mathcal{P}_{\varepsilon}\right)$ strongly converges to some $\bar{u}$ in $L^{1}\left(\Omega, \mathbf{R}^{3}\right)$ whose distributional gradient has no Cantor part, according to the variational nature of the $\Gamma$-convergence, we deduce that $\bar{u}$ is a solution of the limit problem

$$
\min \left\{F_{0}(u)-L(u): u \in L^{1}\left(\Omega, \mathbf{R}^{3}\right)\right\}
$$

and

$$
\inf \left\{F_{\varepsilon}(u)-L(u): u \in L^{1}\left(\Omega, \mathbf{R}^{3}\right)\right\} \rightarrow \min \left\{F_{0}(u)-L(u): u \in \tilde{A}_{0}\right\} .
$$

Therefore, under the asumption that some approximate minimizer is regular in the sense above, problem $(\mathcal{P})$ is a good model in the sense of Section 3, where the density functions are now $Q f$ and $S Q g_{0}$.

Remark 2. In the case when the deformations in the adhesive may be large, they are modeled as hyperelastic together with the deformations in the adherents. 
In this particular case, we obtain a complete description of the $\Gamma$-limit $F_{0}$ in the set

$$
\tilde{A}_{0}^{\prime}:=\left\{u \in W_{\Gamma_{0}}^{1, p}\left(\Omega, \mathbf{R}^{3}\right): \gamma_{S}(u) \in B V\left(S, \mathbf{R}^{3}\right)\right\} .
$$

More precisely $F_{0}$ is defined in $\tilde{A}_{0}^{\prime}$ by

$$
\begin{aligned}
F_{0}(u)=\int_{\Omega} Q f(\nabla u) d x & +\int_{S} Q g_{0}\left(\nabla\left(\gamma_{S}(u)\right)\right) d \hat{x} \\
& +\int_{S}\left(Q g_{0}\right)^{\infty}\left(\frac{D_{s} \gamma_{S}(u)}{\left|D_{s} \gamma_{S}(u)\right|}\right)\left|D_{s} \gamma_{S}(u)\right| d \mathcal{H}^{1},
\end{aligned}
$$

where $D u=\nabla u d \hat{x}+D_{s} u$ is the Lebesgue decomposition of the distributional derivative $D u, g_{0}(\zeta)=\min \left\{g(\xi): \xi \in \mathbf{M}^{3 \times 3}, \hat{\xi}=\zeta\right\}$ for every $\zeta \in \mathbf{R}^{3}$, and $\zeta \mapsto\left(Q g_{0}\right)^{\infty}(\zeta):=\lim _{t \rightarrow+\infty} Q g_{0}(t \zeta) / t$ is the recession function of $Q g_{0}$. The proof uses the relaxation theorem, Theorem 11.3.1 in [6], instead of Proposition 4 below and follows point by point the claims of Propositions 3, 5 below.

Proposition 3 (Lower bound). For all $u$ in $\tilde{A}_{0}$ and all sequence $\left(u_{\varepsilon}\right)_{\varepsilon>0}$ in $L^{1}\left(\Omega, \mathbf{R}^{3}\right)$ such that $u_{\varepsilon} \rightarrow u$, the following inequality holds:

$$
F_{0}(u) \leq \liminf _{\varepsilon \rightarrow 0} F_{\varepsilon}\left(u_{\varepsilon}\right) .
$$

Proof. One has

$$
\begin{aligned}
\liminf _{\varepsilon \rightarrow 0} F_{\varepsilon}\left(u_{\varepsilon}\right) & =\liminf _{\varepsilon \rightarrow 0}\left(\int_{\Omega_{\varepsilon}} f\left(\nabla u_{\varepsilon}\right) d x+\frac{1}{\varepsilon} \int_{B_{\varepsilon}} g\left(e\left(u_{\varepsilon}\right)\right) d x\right) \\
& \geq \liminf _{\varepsilon \rightarrow 0} \int_{\Omega_{\varepsilon}} f\left(\nabla u_{\varepsilon}\right) d x+\liminf _{\varepsilon \rightarrow 0} \frac{1}{\varepsilon} \int_{B_{\varepsilon}} g\left(e\left(u_{\varepsilon}\right)\right) d x \\
& \geq \liminf _{\varepsilon \rightarrow 0} \int_{\Omega_{\varepsilon}} f\left(\nabla u_{\varepsilon}\right) d x+\liminf _{\varepsilon \rightarrow 0} \frac{1}{\varepsilon} \int_{B_{\varepsilon}} g_{0}\left(\widehat{e\left(u_{\varepsilon}\right)}\right) d x
\end{aligned}
$$

For a.e. $x$ in $B$, set $\hat{v}_{\varepsilon}(x):=\hat{v}_{\varepsilon}\left(\hat{x}, \varepsilon x_{3}\right)$ and $v_{\varepsilon}^{3}(x)=\varepsilon v_{\varepsilon}^{3}\left(\hat{x}, x_{3}\right)$. Set $B:=$ $S \times\left(-\frac{1}{2}, \frac{1}{2}\right)$. We have

$$
\liminf _{\varepsilon \rightarrow 0} \frac{1}{\varepsilon} \int_{B_{\varepsilon}} g_{0}\left(\widehat{e\left(u_{\varepsilon}\right)}\right) d x \geq \liminf _{\varepsilon \rightarrow 0} \int_{B} S Q g_{0}\left(\widehat{\left(e\left(v_{\varepsilon}\right)\right.}\right) d x .
$$

Consider the function $h: \mathbf{M}_{s}^{3 \times 3} \rightarrow \mathbf{R}$ defined for every $\xi \in \mathbf{M}_{s}^{3 \times 3}$ by $h(\xi):=$ $S Q g_{0}(\hat{\xi})$. It is easily seen that $h$ is symmetric quasiconvex, i.e. satisfies the inequality:

$$
h(\xi) \leq \frac{1}{|D|} \int_{D} h(\xi+e(\varphi)) d x
$$

for every $\varphi \in \mathcal{C}_{c}^{\infty}\left(D, \mathbf{R}^{3}\right)$ where $D$ is any cube of $\mathbf{R}^{3}$ (see [9] for the definition). Moreover (17) can be written

$$
\liminf _{\varepsilon \rightarrow 0} \frac{1}{\varepsilon} \int_{B_{\varepsilon}} g_{0}\left(\widehat{e\left(u_{\varepsilon}\right)}\right) d x \geq \liminf _{\varepsilon \rightarrow 0} \int_{B} h\left(e\left(v_{\varepsilon}\right)\right) d x .
$$


Let denote by $\operatorname{BD}\left(B, \mathbf{R}^{3}\right)$ the space of bounded deformation on $B$ and by $\mathcal{R}_{H}$ the set of rigid motions on $B$. According to coercivity condition (2) we have

$$
\sup _{\varepsilon>0} \int_{B}\left|\left(\begin{array}{cc}
e_{\alpha \beta}\left(\hat{v}_{\varepsilon}\right) & \frac{1}{\varepsilon} e_{\alpha 3}\left(v_{\varepsilon}\right) \\
\frac{1}{\varepsilon} e_{3 \alpha}\left(v_{\varepsilon}\right) & \frac{1}{\varepsilon^{2}} \frac{\partial v_{3}^{\varepsilon}}{\partial v_{3}}
\end{array}\right)\right| d x \leq \frac{1}{\alpha} \sup _{\varepsilon>0} \frac{1}{\varepsilon} \int_{B_{\varepsilon}} g\left(e\left(u_{\varepsilon}\right)\right) d x<+\infty .
$$

Thus, by using the arguments of the proof of Lemma 2, one can easily establish the existence of $v \in \mathrm{BD}\left(B, \mathbf{R}^{3}\right)$ and $r_{\varepsilon} \in \mathcal{R}_{H}$ such that $v_{\varepsilon}+r_{\varepsilon} \rightarrow v$ in $\mathrm{BD}\left(B, \mathbf{R}^{3}\right), \hat{v}=\gamma_{S}(\hat{u}), v_{3}=0$ and $\frac{\partial v}{\partial x_{3}}=0$. Combining (16), (18), a classical results in relaxation theory (Theorem 11.2.1 in [6]) and a relaxation result in $\mathrm{BD}\left(S, \mathbf{R}^{2}\right)$ (see [9]), we infer

$$
\begin{aligned}
\liminf _{\varepsilon \rightarrow 0} F_{\varepsilon}\left(u_{\varepsilon}\right) \geq & \int_{\Omega} Q f(\nabla u) d x+\int_{S} S Q g_{0}\left(e_{a}\left(\gamma_{S}(\hat{u})\right) d \hat{x}\right. \\
& +\int_{S} S Q g_{0}\left(\left[\gamma_{s}(\hat{u})\right] \otimes_{s} \nu_{\gamma_{S}(u)}(\hat{x})\right) d \mathcal{H}^{1}(\hat{x})
\end{aligned}
$$

which ends the proof.

For proving the upper bound, we need to establish the following relaxation result.

Proposition 4 (Relaxation). The functional $F_{0}$ is the l.s.c. regularization for strong topology of the functional defined on $L^{1}\left(\Omega, \mathbf{R}^{3}\right)$ by

$$
\tilde{F}_{0}(u):=\left\{\begin{array}{l}
\int_{\Omega} f(\nabla u) d x+\int_{S} g_{0}\left(e\left(\gamma_{S}(\hat{u})\right) d \hat{x} \text { if } u \in A_{0}^{1},\right. \\
+\infty \text { otherwise. }
\end{array}\right.
$$

In the proof of Proposition 4 we will use the following lemma.

Lemma 4. Let $\eta>0$, then for every $\zeta \in \mathbf{M}_{s}^{2 \times 2}$,

$$
\lim _{\eta \rightarrow 0} S Q\left(\eta|\cdot|^{p}+g_{0}\right)(\zeta)=S Q g_{0}(\zeta) .
$$

Proof. From the integral representations

$$
\left.S Q\left(\eta|\cdot|^{p}+g_{0}\right)(\zeta)=\inf _{\phi \in C_{c}^{\infty}\left(D, \mathbf{R}^{2}\right)} \frac{1}{|D|} \int_{D}\left(\eta|\cdot|^{p}+g_{0}\right)\left(\zeta+e_{(} \phi\right)\right) d \hat{x},
$$

and

$$
S Q g_{0}(\zeta)=\inf _{\phi \in C_{c}^{\infty}\left(D, \mathbf{R}^{2}\right)} \frac{1}{|D|} \int_{D} g_{0}(\zeta+e(\phi)) d \hat{x}
$$


where $D$ is an arbitrary cube of $\mathbf{R}^{2}$, we can write

$$
\begin{aligned}
& \lim _{\eta \rightarrow 0}\left(\inf _{\phi \in C_{c}^{\infty}\left(D, \mathbf{R}^{2}\right)} \frac{1}{|D|} \int_{D}\left(\eta|\cdot|^{p}+g_{0}\right)(\zeta+e(\phi)) d \hat{x}\right) \\
= & \inf _{\eta>0}\left(\inf _{\phi \in C_{c}^{\infty}\left(D, \mathbf{R}^{2}\right)} \frac{1}{|D|} \int_{D}\left(\eta|\cdot|^{p}+g_{0}\right)(\zeta+e(\phi)) d \hat{x}\right) \\
= & \inf _{\phi \in C_{c}^{\infty}\left(D, \mathbf{R}^{2}\right)}\left(\inf _{\eta>0} \frac{1}{|D|} \int_{D}\left(\eta|\cdot|^{p}+g_{0}\right)(\zeta+e(\phi)) d \hat{x}\right) \\
= & \inf _{\phi \in C_{c}^{\infty}\left(D, \mathbf{R}^{2}\right)}\left(\lim _{\eta \rightarrow 0} \frac{1}{|D|} \int_{D}\left(\eta|\cdot|^{p}+g_{0}\right)(\zeta+e(\phi)) d \hat{x}\right) .
\end{aligned}
$$

We complete the proof by using the dominated convergence theorem.

Proof of Proposition 4. Denote by $\overline{\tilde{F}}_{0}$ the l.s.c. regularization of $\tilde{F}_{0}$. By using standard l.s.c. results on integral functionals defined on Sobolev or $B V$-spaces, one can easily prove $F_{0} \leq \overline{\tilde{F}}_{0}$ (see for instance [6], chapter 10). We only establish the converse inequality $\overline{\tilde{F}}_{0} \leq F_{0}$. Its proof is not easy because of the condition $u \in A_{0}$ and the fact that $f$ and $g$ do not fulfill the same growth conditions.

Let $u \in L^{1}\left(\Omega, \mathbf{R}^{3}\right)$ such that $F_{0}(u)<+\infty$. Then $u \in A_{0}$. We have to exhibit $u_{n}$ in $A_{0}^{1}$ strongly converging to $u$ in $L^{1}\left(\Omega, \mathbf{R}^{3}\right)$ such that $\lim _{n \rightarrow+\infty} \tilde{F}_{0}\left(u_{n}\right)=$ $F_{0}(u)$. We proceed into two steps.

Step 1. We prove the thesis when $u \in A_{0}^{1}$. For shorten notation we write $v:=\gamma_{S}(\hat{u})$.

Let $\eta>0$ intended to go to 0 and denote the constant involved in Korn's inequality by $K$ :

$$
\int_{S}|\nabla w|^{p} d \hat{x} \leq K\left(\int_{S}|e(w)|^{p}+|w|^{p}\right) d \hat{x}
$$

for all function $w$ in $W^{1, p}\left(S, \mathbf{R}^{2}\right)$. From relaxation theory in Sobolev spaces, there exists a sequence of smooth functions $\left(u_{n}, v_{n}\right)_{n \in \mathbf{N}}$ in $W_{\Gamma_{0}}^{1, p}\left(\Omega, \mathbf{R}^{3}\right) \times W^{1, p}\left(S, \mathbf{R}^{2}\right)$ such that (see for instance [6], Theorem 11.2.1 and Theorem 11.4.2)

$$
\left\{\begin{array}{l}
\left.u_{n} \rightarrow u \text { in } W_{\Gamma_{0}^{1, p}}^{1, \Omega}, \mathbf{R}^{3}\right),\left(\left|\nabla u_{n}\right|^{p}\right)_{n \in \mathbf{N}} \text { uniformly integrable; } \\
v_{n} \rightarrow v \text { in } W^{1, p}\left(S, \mathbf{R}^{3}\right) ; \\
\lim _{n \rightarrow+\infty} \int_{\Omega} f\left(\nabla u_{n}\right) d x=\int_{\Omega} Q f(\nabla u) d x \\
\lim _{n \rightarrow+\infty} \int_{S}\left(3 K \beta \eta|\cdot|^{p}+g_{0}\right)\left(e\left(v_{n}\right)\right) d \hat{x}=\int_{S} S Q\left(3 K \beta \eta|\cdot|^{p}+g_{0}\right)(e(v)) d \hat{x} .
\end{array}\right.
$$

The additional condition that $\left(\left|\nabla u_{n}\right|^{p}\right)_{n \in \mathbf{N}}$ may be assumed to be uniformly integrable comes from the following consideration. Consider the sequence $\left(\tilde{u}_{n}\right)_{n \in \mathbf{N}}$ whose gradients generate the same Young measure $\mu$ and such that $\left(\left|\nabla \tilde{u}_{n}\right|^{p}\right)_{n \in \mathbf{N}}$ is unifomly integrable (Lemma 11.4.1 in [6]). By using lower semicontinuity and 
continuity properties of Young measures (Proposition 4.3.3 and Theorem 4.3.3 in [6]), and standard lower semicontinuity results in Sobolev spaces, we have

$$
\begin{aligned}
\int_{\Omega} \mathcal{Q} f(\nabla u) d x & =\lim _{n \rightarrow+\infty} \int_{\Omega} f\left(\nabla u_{n}\right) d x \\
& \geq \int_{\Omega \times \mathbf{M}^{3 \times 3}} f(\lambda) d \mu \\
& =\lim _{n \rightarrow+\infty} \int_{\Omega} f\left(\nabla \tilde{u}_{n}\right) d x \\
& \geq \int_{\Omega} \mathcal{Q} f(\nabla u) d x
\end{aligned}
$$

so that

$$
\lim _{n \rightarrow+\infty} \int_{\Omega} f\left(\nabla \tilde{u}_{n}\right) d x=\lim _{n \rightarrow+\infty} \int_{\Omega} f\left(\nabla u_{n}\right) d x=\int_{\Omega} \mathcal{Q} f(\nabla u) d x
$$

which proves the thesis. In what follows, we still denote by $\left(u_{n}\right)_{n \in \mathbf{N}}$ the sequence $\left(\tilde{u}_{n}\right)_{n \in \mathbf{N}}$.

We start by modifying the function $u_{n}$ near $S$ so that $\gamma_{S}\left(u_{\alpha}^{n}\right)=v_{\alpha}^{n}$. Set $\Sigma_{\eta}:=S \times(-\eta, \eta), \Sigma_{2 \eta}:=S \times(-2 \eta, 2 \eta)$, consider a cut-off function $\varphi_{\eta}$ in $\mathcal{C}^{1}(\mathbf{R})$ satisfying

$$
\varphi_{\eta}=1 \text { on } \Omega \backslash \Sigma_{2 \eta}, \varphi_{\eta}=0 \text { on } \Sigma_{\eta}, 0 \leq \varphi_{\eta} \leq 1,\left|\frac{d \varphi_{\eta}}{d x_{3}}\right| \leq \frac{1}{\eta},
$$

and define the function $u_{n, \eta}$ by

$$
\begin{aligned}
& u_{\alpha}^{n, \eta}:=\varphi_{\eta}\left(u_{\alpha}^{n}-v_{\alpha}^{n}\right)+v_{\alpha}^{n} \\
& u_{3}^{n, \eta}:=u_{3}^{n} .
\end{aligned}
$$

Clearly $u_{n, \eta} \in W_{\Gamma_{0}}^{1, p}\left(\Omega, \mathbf{R}^{3}\right)$ and $\gamma_{S}\left(u_{\alpha}^{n, i}\right)=v_{\alpha}^{n}$. From the growth condition satisfied by $f$, we have

$$
\begin{aligned}
\int_{\Omega} f\left(\nabla u_{n, \eta}\right) d x & =\int_{\Sigma_{\eta}} f\left(\nabla u_{n, \eta}\right) d x+\int_{\Sigma_{2 \eta} \backslash \Sigma_{\eta}} f\left(\nabla u_{n, \eta}\right) d x+\int_{\Omega \backslash \Sigma_{2 \eta}} f\left(\nabla u_{n}\right) d x \\
\leq & \beta \int_{\Sigma_{\eta}}\left(1+\left|\nabla v_{n}\right|^{p}\right) d x+\beta \int_{\Sigma_{2 \eta} \backslash \Sigma_{\eta}}\left(1+\left|\nabla v_{n}\right|^{p}\right) d x+C\left[\int_{\Sigma_{2 \eta}}\left|\nabla u_{n}\right|^{p} d x\right. \\
& \left.+\frac{1}{\eta^{p}} \int_{\Sigma_{2 \eta}}\left|\hat{u}_{n}-v_{n}\right|^{p} d x\right]+\int_{\Omega} f\left(\nabla u_{n}\right) d x \\
= & 3 \beta \eta \int_{S}\left|\nabla v_{n}\right|^{p} d \hat{x}+C\left[\eta+\int_{\Sigma_{2 \eta}}\left|\nabla u_{n}\right|^{p} d x+\frac{1}{\eta^{p}} \int_{\Sigma_{2 \eta}}\left|\hat{u}_{n}-v_{n}\right|^{p} d x\right] \\
& +\int_{\Omega} f\left(\nabla u_{n}\right) d x .
\end{aligned}
$$


Thus, according to Korn's inequality (note that $\sup _{n \in \mathbf{N}} \int_{S}\left|v_{n}\right|^{p} d \hat{x}<+\infty$ ),

$$
\begin{aligned}
\int_{\Omega} f\left(\nabla u_{n, \eta}\right) d x & \leq 3 \beta K \eta \int_{S} \mid\left(\left.e\left(v_{n}\right)\right|^{p} d \hat{x}\right. \\
& +C\left[\eta+\int_{\Sigma_{2 \eta}}\left|\nabla u_{n}\right|^{p} d x+\frac{1}{\eta^{p}} \int_{\Sigma_{2 \eta}}\left|\hat{u}_{n}-v_{n}\right|^{p} d x\right]+\int_{\Omega} f\left(\nabla u_{n}\right) d x .
\end{aligned}
$$

which yields

$$
\begin{aligned}
\int_{\Omega} f\left(\nabla u_{n, \eta}\right) d x+\int_{S} g_{0}\left(e\left(v_{n}\right)\right) d \hat{x} \leq & \int_{\Omega} f\left(\nabla u_{n}\right) d x+\int_{S}\left(3 \beta K \eta||^{p}+g_{0}\right)\left(e\left(v_{n}\right)\right) d \hat{x} \\
& +C\left[\eta+\int_{\Sigma_{2 \eta}}\left|\nabla u_{n}\right|^{p} d x+\frac{1}{\eta^{p}} \int_{\Sigma_{2 \eta}}\left|\hat{u}_{n}-v_{n}\right|^{p} d x\right]
\end{aligned}
$$

and, from (4),

$$
\begin{aligned}
\limsup _{n \rightarrow+\infty} \tilde{F}_{0}\left(u_{n, \eta}\right) \leq & \int_{\Omega} Q f(\nabla u) d x+\int_{S} S Q\left(3 \beta K \eta||^{p}+g_{0}\right)\left(e\left(v_{n}\right)\right) d \hat{x} \\
& +C\left[\eta+\sup _{n \in \mathbf{N}} \int_{\Sigma_{2 \eta}}\left|\nabla u_{n}\right|^{p} d x+\frac{1}{\eta^{p}} \int_{\Sigma_{2 \eta}}|\hat{u}-v|^{p} d x\right]
\end{aligned}
$$

But since $\gamma_{S}(\hat{u})=v$, clearly one has

$$
\int_{\Sigma_{2 \eta}}|\hat{u}-v|^{p} d x \leq \eta^{p} \int_{\Sigma_{2 \eta}}\left|\frac{\partial u}{\partial x_{3}}\right|^{p} d x
$$

so that

$$
\begin{aligned}
\limsup _{n \rightarrow+\infty} \tilde{F}_{0}\left(u_{n, \eta}\right) \leq & \left.\int_{\Omega} Q f(\nabla u) d x+\int_{S} S Q\left(3 \beta K \eta||^{p}+g_{0}\right) e(v)\right) d \hat{x} \\
& +C\left[\eta+\sup _{n \in \mathbf{N}} \int_{\Sigma_{2 \eta}}\left|\nabla u_{n}\right|^{p} d x+\int_{\Sigma_{2 \eta}}\left|\frac{\partial u}{\partial x_{3}}\right|^{p} d x\right]
\end{aligned}
$$

By letting $\eta \rightarrow 0$, from the uniform integrability of $\left(\left|\nabla u_{n}\right|^{p}\right)_{n \in \mathbf{N}}$, Lemma 4 and Lebesgue's dominated convergence theorem, we obtain

$$
\limsup _{\eta \rightarrow 0} \limsup _{n \rightarrow+\infty} \tilde{F}_{0}\left(u_{n, \eta}\right) \leq \int_{\Omega} Q f(\nabla u) d x+\int_{S} S Q g_{0}(e(v)) d \hat{x} .
$$

By using a standard diagonalization argument, there exists a map $n \mapsto \eta(n)$ such that, setting $\tilde{u}_{n}:=u_{n, \eta(n)}$,

$$
\limsup _{n \rightarrow+\infty} \tilde{F}_{0}\left(\tilde{u}_{n}\right) \leq F_{0}(u) .
$$

It is easily seen that $\tilde{u}_{n} \rightarrow u$ in $L^{1}\left(\Omega, \mathbf{R}^{3}\right)$. Since classically $\lim \inf _{n \rightarrow+\infty} \tilde{F}_{0}\left(\tilde{u}_{n}\right) \geq$ $F_{0}(u)$, the proof of step 1 is complete. 
Step 2. We end the proof as the step 2 of the proof of Lemma 3. We only have to substitute

$$
\mid \int_{\mathbf{R}^{2}} S Q g_{0}\left(\rho_{\eta(\delta)} *(\phi e(\tilde{v})) d \hat{x}-\int_{\mathbf{R}^{2}} S Q g_{0}(\phi e(v) \mid<\delta\right.
$$

for (10). Estimate (20) is a straightforward consequence of the weak convergence of the measure $\left.\rho_{\eta(\delta)} *(\phi e(v))\right)$ to the measure $\left.\phi e(v)\right)$ in $\mathbf{M}\left(\mathbf{R}^{2}\right)$ together with $\left.\left.\lim _{n \rightarrow+\infty} \int_{\mathbf{R}^{2}} \mid \rho_{\eta(\delta)} *(\phi e(v))\right)\left|=\int_{\mathbf{R}^{2}}\right| \phi e(v)\right) \mid$, and Reshetnyak's continuity theorem (see Theorem 2.39 in [4]).

Then proceeding exactly like in the proof of Proposition 2 and using Lemma 4 , one has

Proposition 5 (Upper bound). The following inequality holds in $L^{1}\left(\Omega, \mathbf{R}^{3}\right)$

$$
\left(\Gamma-\lim \sup F_{\varepsilon}\right) \leq F_{0} .
$$

\section{A modeling of a welding assembly}

An elementary situation in welding can be described as follows. Let $\Sigma^{+}, \Sigma^{-}$ and $S$ three domains of $\mathbf{R}^{2}$ with Lipschitz-continuous boundaries such that $S=\Sigma^{+} \cap \Sigma^{-}$. Let $r$ and $\varepsilon$ two positive numbers such that $\varepsilon<<r$ and $\Omega_{\varepsilon}^{ \pm}:=$ $\Sigma^{ \pm} \times( \pm \varepsilon / 2, \pm r), \Omega_{\varepsilon}=\Omega_{\varepsilon}^{+} \cup \Omega_{\varepsilon}^{-}, B_{\varepsilon}=S \times(-\varepsilon / 2, \varepsilon / 2)$, and $S_{\varepsilon}^{ \pm}=S^{ \pm} \pm \varepsilon / 2 e_{3}$. Then $\mathcal{O}_{\varepsilon}:=\Omega_{\varepsilon} \cup S_{\varepsilon}^{+} \cup S_{\varepsilon}^{-} \cup B_{\varepsilon}$ is the reference configuration of a structure made of two adherents and an adhesive (the soldered joint) which respectively occupies $\Omega_{\varepsilon}^{ \pm}$and $B_{\varepsilon}$ (see Figure 1 ). The structure is clamped on a part $\Gamma_{0}$ of the boundary $\Gamma$ of $\Omega$ with a positive $\mathcal{H}^{2}$-measure and we assume that there exists $\varepsilon_{0}>0$ such that $\operatorname{dist}\left(\bar{\Gamma}_{0}, \bar{B}_{\varepsilon_{0}}\right)>0$. The structure is subjected to body forces of density $\Phi$ and to surface forces of density $\varphi$ on the complementary part $\Gamma_{\varphi}$ of $\Gamma_{0}$. We assume that the supports of $\varphi$ and $\Phi$ lay outside of $\bar{B}_{\varepsilon_{0}}$. Obviously one can consider other type of boundary conditions (e.g. a combination of some components of the stress vector and of the displacement). At last, adhesive and adherents are assumed to be perfectly stuck together along $S^{ \pm}$.

The adherents and the adhesive are modeled as in section 2 so that determining the equilibrium configuration leads to the problem

$$
\inf \left\{F_{\varepsilon}(u)-L(u): u \in A_{\varepsilon}\right\}
$$

where $F_{\varepsilon}$ and $L$ have the same expression as in Section 2 but with the new definitions of $\Omega_{\varepsilon}$ and $B_{\varepsilon}$, whereas $A_{\varepsilon}$ now reads as:

$$
A_{\varepsilon}:=\left\{u \in \operatorname{LD}\left(\mathcal{O}_{\varepsilon}, \mathbf{R}^{3}\right): u_{\left\lfloor\Omega_{\varepsilon}\right.} \in W_{\Gamma_{0}}^{1, p}\left(\Omega_{\varepsilon}, \mathbf{R}^{3}\right)\right\} .
$$

Again, to propose a simplified but accurate model we consider $\varepsilon$ as a parameter and study the asymptotic behavior, when $\varepsilon$ goes to zero, of (approximate) solution of $\left(\mathcal{P}_{\varepsilon}\right)$. The essential difference from the model problem of Section 2 is that here the structure occupies a domain $\mathcal{O}_{\varepsilon}$ which varies with $\varepsilon$, which from 


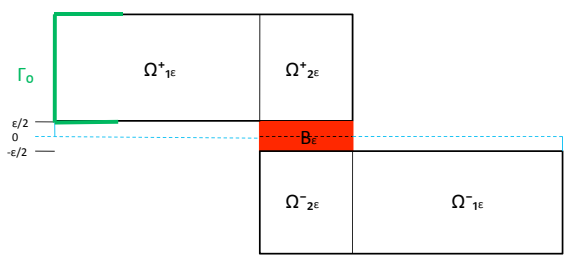

Figure 1: the reference configuration $\mathcal{O}_{\varepsilon}$. The reference configuration involved in Section 2 is $\Omega_{2 \varepsilon}^{+} \cup \Omega_{2 \varepsilon}^{-} \cup B_{\varepsilon} \cup S_{\varepsilon}^{+} \cup S_{\varepsilon}^{-}$.

the mathematical point of view is only of technical nature by simply modifying the kinds of convergences. That is why we have preferred to consider the model problem in whole details and to confine to state the sole results about this realistic problem of welding.

Let $\Omega:=\Sigma^{+} \times(0, r) \cup S \cup \Sigma^{-} \times(-r, 0)$ the set to which $\mathcal{O}_{\varepsilon}$ "converges". Let $I_{\varepsilon}:=F_{\varepsilon}-L$ and $I_{0}:=F_{0}-L$ with $F_{0}: L^{1}\left(\Omega, \mathbf{R}^{3}\right) \rightarrow \mathbf{R} \cup\{+\infty\}$,

$$
F_{0}(u):=\left\{\begin{array}{l}
\int_{\Omega} f(\nabla u) d x+\int_{S}\left(g _ { 0 } \left(e\left(\gamma_{s}(\hat{u})\right) \text { if } u \in A_{0}\right.\right. \\
+\infty \text { otherwise, }
\end{array}\right.
$$

where we keep the same definition for $\gamma_{S}$ and $A_{0}$ but with the new definition of $\Omega$. Doing the same for the definition of the operator $T_{\varepsilon}$, our asymptotic model is supplied by:

Theorem 3. Let $\bar{u}_{\varepsilon}$ be a solution of $\left(\mathcal{P}_{\varepsilon}\right)$. Then there exist a subsequence of $\left(\bar{u}_{\varepsilon}\right)_{\varepsilon>0}$ and $\bar{u}$ in $W_{\Gamma_{0}}^{1, p}\left(\Omega, \mathbf{R}^{3}\right)$ such that

$$
\begin{aligned}
& T_{\varepsilon} \bar{u}_{\varepsilon} \rightarrow \bar{u} \text { weakly in } W^{1, p}\left(\Omega \backslash S, \mathbf{R}^{3}\right) \text {; } \\
& \bar{u}_{\varepsilon\lfloor S \times(-r, r)} \rightarrow \bar{u} \text { weakly in } B D\left(S \times(-r, r), \mathbf{R}^{3}\right) \text {; } \\
& \gamma_{S}(\hat{\bar{u}}) \in B D\left(S, \mathbf{R}^{2}\right) \text {. }
\end{aligned}
$$

Moreover $\bar{u}$ is solution of the minimization problem

$$
\min \left\{F_{0}(u)-L(u): u \in L^{1}\left(\Omega, \mathbf{R}^{3}\right)\right\}
$$

and

$$
\min \left\{F_{\varepsilon}(u)-L(u): u \in L^{1}\left(\Omega, \mathbf{R}^{3}\right)\right\} \rightarrow \min \left\{F_{0}(u)-L(u): u \in L^{1}\left(\Omega, \mathbf{R}^{3}\right)\right\} .
$$


Sketch of the proof. The proof follows the line of the proof of Section 2 by considering simultaneously $T_{\varepsilon} \bar{u}_{\varepsilon}$ and its restriction to the fixed domain $S \times$ $(-r, r)$.

For the mechanical interpretation see the end of Section 3.

\section{A variational regularization of the limit func- tional}

For the numerical solving of the optimization problem

$$
\inf \left\{F_{0}(u)-L(u)\right\}
$$

obtained in Section 3, we approximate, in a variational way, the functional of measure $u \mapsto \int_{S} g_{0}\left(e\left(\gamma_{S}(\hat{u})\right)\right.$ by a suitable functional defined in the Sobolev space $W^{1, q}\left(S, \mathbf{R}^{2}\right)$, where $q$ is close to 1 in the spirit of Norton-Hoff regularisation (cf [17]). The mathematical technics used here is an adaptation from that of [7]. In order to simplify the proofs, we assume that $S$ is a finite union of cubes in $\mathbf{R}^{2}$.

We denote the limit density $g_{0}$ by $h$ that we assume to be positively 1 homogeneous and fulfilling the growth conditions $\alpha|\xi| \leq h(\xi) \leq \beta|\xi|$. We consider a sequence $\left(h_{q}\right)_{q \in(1, p)}$ satisfying the following three conditions:

$\left.i_{q}\right) h_{q}: \mathbf{M}_{s}^{2 \times 2} \rightarrow \mathbf{R}^{+}$is convex and positively homogeneous of degree $q$;

$\left.i i_{q}\right) h_{q} \rightarrow h$ pointwise in $\mathbf{M}_{s}^{2 \times 2}$;

$i i i_{q}$ ) there exists $a>0$ such that for all $q>1$ close enough to 1 ,

$$
h_{q}(\xi) \geq h(\xi) \text { for all } \xi \in \mathbf{M}_{s}^{2 \times 2},|\xi| \geq a .
$$

For instance, when $h=||,. h_{q}=|.|^{q}$ satisfies these conditions with $a=1$. Another natural example consists in choosing $h_{q}:=h^{q}$. Condition $i i i_{q}$ ) is then satisfyed by taking $a=\frac{1}{\alpha}$. In these two examples, $h_{q}$ satisfies uniform growth conditions with respect to $q$ (for the second example, $\frac{\alpha}{2}|\xi|^{q} \leq h_{q}(\xi) \leq 2 \beta|\xi|^{q}$

for $q$ closed to 1 ). Note that, according to $i i i)_{q}, h_{q}$ fulfills the equi-coerciveness condition:

$$
h_{q}(\xi) \geq \alpha|\xi| \forall \xi,|\xi| \geq a .
$$

In what follows, the function $h_{q}$ is not assumed to satisfy a uniform upper growth condition.

We consider the functional $\mathcal{F}_{q}: W_{\Gamma_{0}}^{1, p}\left(\Omega, \mathbf{R}^{3}\right) \rightarrow \mathbf{R}^{+} \cup\{+\infty\}$ defined by:

$$
\mathcal{F}_{q}(u)=\left\{\begin{array}{l}
\int_{\Omega} f(\nabla u) d x+\int_{S} h_{q}\left(e\left(\gamma_{S}(\hat{u})\right)\right) d \hat{x} \text { if } u \in \mathcal{B}_{q}, \\
+\infty \text { otherwise }
\end{array}\right.
$$


where

$$
\mathcal{B}_{q}:=\left\{u \in W_{\Gamma_{0}}^{1, p}\left(\Omega, \mathbf{R}^{3}\right): h_{q} \circ\left(e\left(\gamma_{S}(\hat{u})\right)\right) \in L^{1}(\Omega)\right\} .
$$

We are going to establish the $\Gamma$-convergence of $\mathcal{F}_{q}$ when $q \rightarrow 1$, when the space $W_{\Gamma_{0}}^{1, p}\left(\Omega, \mathbf{R}^{3}\right)$ is equipped with its weak topology. The expected limit is the functional $\mathcal{F}_{0}: W_{\Gamma_{0}}^{1, p}\left(\Omega, \mathbf{R}^{3}\right) \rightarrow \mathbf{R}^{+} \cup\{+\infty\}$ defined in the previous section, more precisely its restriction to $W_{\Gamma_{0}}^{1, p}\left(\Omega, \mathbf{R}^{3}\right)$ defined by

$$
\mathcal{F}_{0}(u)=\left\{\begin{array}{l}
\int_{\Omega} f(\nabla u) d x+\int_{S} h\left(e\left(\gamma_{S}(\hat{u})\right)\right) \text { if } u \in \mathcal{B} \\
+\infty \text { otherwise, }
\end{array}\right.
$$

where

$$
\mathcal{B}:=\left\{u \in W_{\Gamma_{0}}^{1, p}\left(\Omega, \mathbf{R}^{3}\right): \gamma_{S}(\hat{u}) \in \operatorname{BD}\left(S, \mathbf{R}^{2}\right)\right\} .
$$

Lemma 5 (Compactness lemma). Consider a sequence $\left(u_{q}\right)_{q \in] 1, p]}$ in $W_{\Gamma_{0}}^{1, p}\left(\Omega, \mathbf{R}^{3}\right)$ such that $\sup _{q \in(1, p)} \mathcal{F}_{q}\left(u_{q}\right)<+\infty$. Then there exist a subsequence of $\left(u_{q}\right)_{q \in] 1, p]}$ and $u \in \mathcal{B}$ such that $\left(u_{q}, \gamma_{S}\left(\hat{u}_{q}\right)\right) \rightarrow\left(u, \gamma_{S}(\hat{u})\right)$ in $W_{\Gamma_{0}}^{1, p}\left(\Omega, \mathbf{R}^{3}\right) \times B D\left(S, \mathbf{R}^{2}\right)$ when $q$ goes to 1.

Proof. Since $\sup _{q \in(1, p)} \mathcal{F}_{q}\left(u_{q}\right)<+\infty$, one has $u_{q} \in \mathcal{B}_{q}$ and

$$
\left.\mathcal{F}_{q}\left(u_{q}\right)=\int_{\Omega} f\left(\nabla u_{q}\right)\right) d x+\int_{S} h_{q}\left(e\left(v_{q}\right) d \hat{x}, v_{q}:=\gamma_{S}\left(\hat{u}_{q}\right) .\right.
$$

Thus, from (23)

$$
\begin{aligned}
\sup _{q \in(1, p)} \int_{S}\left|e\left(v_{q}\right)\right| d \hat{x} & \leq \sup _{q \in(1, p)}\left(a|S|+\int_{\left[\left|e\left(v_{q}\right)\right| \geq a\right]}\left|e\left(v_{q}\right)\right| d \hat{x}\right) \\
& \leq a|S|+\frac{1}{\alpha} \sup _{q \in(1, p)} \int_{S} h_{q}\left(e\left(v_{q}\right)\right) d \hat{x}<+\infty .
\end{aligned}
$$

On the other hand from the coercivity condition fulfilled by $f$, there exists a subsequence and $u \in W_{\Gamma_{0}}^{1, p}\left(\Omega, \mathbf{R}^{3}\right)$ such that $u_{q} \rightarrow u$ in $W_{\Gamma_{0}}^{1, p}\left(\Omega, \mathbf{R}^{3}\right)$. According to the continuity of the trace operator $\gamma_{S}$, we deduce that $v_{q} \rightarrow \gamma_{S}(\hat{u})$ in $L^{p}\left(S, \mathbf{R}^{3}\right)$, thus strongly in $L^{1}\left(S, \mathbf{R}^{2}\right)$ which, combined with (24), yields $v_{q} \rightarrow v=\gamma_{S}(\hat{u})$ in $\operatorname{BD}\left(S, \mathbf{R}^{2}\right)$ and $u \in \mathcal{B}$.

Proposition 6 (lower bound). For every sequence $\left(\left(u_{q}\right)_{q \in] 1, p]}\right.$ converging to $u$ in $W_{\Gamma_{0}}^{1, p}\left(\Omega, \mathbf{R}^{3}\right)$, we have

$$
\mathcal{F}_{0}(u) \leq \liminf _{q \rightarrow 1} \mathcal{F}_{q}\left(u_{q}\right) .
$$

Lemma 6. Let $V_{q} \in L^{q}\left(S, \mathbf{M}_{s}^{2 \times 2}\right)$ and assume that the measure $\mu_{q}=V_{q} d \hat{x}$ weakly converges to $\mu$ in $\mathcal{M}\left(S, \mathbf{M}_{s}^{2 \times 2}\right)$ and that $\left|\mu_{q}\right|=\left|V_{q}\right| d \hat{x}$ weakly converges to $\nu$ in $\mathcal{M}^{+}(S)$. Then for all open subset $\omega$ of $S$ such that $\nu(\partial \omega)=0$, one has

$$
\liminf _{q \rightarrow 1} \int_{\omega} h_{q}\left(V_{q}\right) d \hat{x} \geq h\left(\int_{\omega} d \mu\right) .
$$


Proof. From Jensen's inequality and since $h_{q}$ is positively $q$-homogeneous,

$$
\int_{\omega} h_{q}\left(V_{q}\right) d \hat{x} \geq|\omega| h_{q}\left(\frac{1}{|\omega|} \int_{\omega} V_{q} d \hat{x}\right)=|\omega|^{1-q} h_{q}\left(\int_{\omega} V_{q} d \hat{x}\right) .
$$

It remains to establish

$$
\liminf _{q \rightarrow 1} h_{q}\left(\int_{\omega} V_{q} d \hat{x}\right) \geq h\left(\int_{\omega} d \mu\right) .
$$

One may assume $\left|\int_{\omega} d \mu\right|>0$, otherwise $\int_{\omega} d \mu=0$ and (25) is trivially satisfied. Since $\nu(\partial \omega)=0$, one has $\lim _{q \rightarrow 1} \int_{\omega} V_{q} d \hat{x}=\int_{\omega} d \mu$ (cf Corollary 4.2.1 in [6]). Then for $0<\sigma<\left|\int_{\omega} d \mu\right|$, there exists $q_{0}, 1<q_{0} \leq p$ such that, for all $q$, $1<q \leq q_{0},\left|\int_{\omega} V_{q} d \hat{x}\right| \geq \sigma$. One has

$$
h_{q}\left(\int_{\omega} V_{q} d \hat{x}\right)=\left(\frac{\sigma}{a}\right)^{q} h_{q}\left(\left(\frac{a}{\sigma} \int_{\omega} V_{q} d \hat{x}\right)\right.
$$

with $\left|\frac{a}{\sigma} \int_{\omega} V_{q} d \hat{x}\right| \geq a$, so that from assumption (22),

$$
\begin{aligned}
h_{q}\left(\int_{\omega} V_{q} d \hat{x}\right) & \geq\left(\frac{\sigma}{a}\right)^{q} h\left(\left(\frac{a}{\sigma} \int_{\omega} V_{q} d \hat{x}\right)\right. \\
& =\left(\frac{\sigma}{a}\right)^{q-1} h\left(\left(\int_{\omega} V_{q} d \hat{x}\right) .\right.
\end{aligned}
$$

Letting $q \rightarrow 1$, (25) follows from the lower semicontinuity of $h$ and the fact that $\lim _{q \rightarrow 1} \int_{\omega} V_{q} d \hat{x}=\int_{\omega} d \mu$.

Proof of Proposition 6. One may assume $\mathcal{F}_{q}\left(u_{q}\right)<+\infty$ so that, from Lemma 5 , one has $u \in \mathcal{B}$. We write $v_{q}$ for $\gamma_{S}\left(\hat{u}_{q}\right)$ and $v$ for $\gamma_{S}(\hat{u})$. According to a standard lower semicontinuity result in Sobolev spaces we have

$$
\liminf _{q \rightarrow 1} \mathcal{F}_{q}\left(u_{q}\right) \geq \int_{\Omega} f(\nabla u) d x+\liminf _{q \rightarrow 1} \int_{S} h_{q}\left(e\left(v_{q}\right)\right) d \hat{x}
$$

and it remains to establish

$$
\liminf _{q \rightarrow 1} \int_{S} h_{q}\left(e\left(v_{q}\right)\right) d \hat{x} \geq \int_{S} h(e(v)) .
$$

For $\delta>0$ intended to go to 0 , consider a standard mollifier $\rho_{\delta}$ and $\theta_{\delta}$ in $\mathcal{C}_{c}^{\infty}(S)$ satisfying $0 \leq \theta_{\delta} \leq 1, \theta_{\delta} \rightarrow 1$ a.e. in $S$. For a subsequence not relabelled on $q$, clearly $\rho_{\delta} * \theta_{\delta}\left|e\left(v_{q}\right)\right| d \hat{x}$ weakly converges to some measure $\nu_{\delta}$ in $\mathcal{M}^{+}(S)$. Moreover $\rho_{\delta} * \theta_{\delta} e\left(v_{q}\right) d \hat{x}$ weakly converges to the measure $\rho_{\delta} * \theta_{\delta} e(v)$ in $\mathcal{M}\left(S, \mathbf{M}_{s}^{2 \times 2}\right)$. For $\eta>0$, consider a finite family $\left(\omega_{i}\right)_{i \in I_{\eta}}$ of pairwise disjoint open subsets of $S,\left|\omega_{i}\right|<\eta$, such that $\left|S \backslash \cup_{i \in I_{\eta}} \omega_{i}\right|=0$ and a family $\left(\tilde{\omega}_{\eta}\right)_{i \in I_{\eta}}$ satisfying

$$
\begin{aligned}
& \left|S \backslash \bigcup_{i \in I_{\eta}} \tilde{\omega}_{i}\right| \leq \eta ; \\
& \tilde{\omega}_{i} \subset \omega_{i} ; \\
& \nu_{\delta}\left(\partial \tilde{\omega}_{i}\right)=0 .
\end{aligned}
$$


Such a family exists (use Lemma 4.2.1 of [6]). Note that this family depends on $\delta$. By using the $q$-homogeneity of $h_{q}$, Jensen's inequality and standard convex duality principle, we have

$$
\begin{aligned}
\int_{S} h_{q}\left(e\left(v_{q}\right)\right) d \hat{x} & \geq \int_{S} \theta_{\delta}^{q} h_{q}\left(e\left(v_{q}\right)\right) d \hat{x} \\
& =\int_{S} h_{q}\left(\theta_{\delta} e\left(v_{q}\right)\right) d \hat{x} \\
& \geq \int_{S} h_{q}\left(\rho_{\delta} *\left(\theta_{\delta} e\left(v_{q}\right)\right) d \hat{x}\right. \\
& \geq \sum_{i \in I_{\eta}} \int_{\tilde{\omega}_{i}} h_{q}\left(\rho_{\delta} *\left(\theta_{\delta} e\left(v_{q}\right)\right)\right) d \hat{x} .
\end{aligned}
$$

Thus, from Lemma 6, 1-homogeneity and Lipschitz property of $h$,

$$
\begin{aligned}
& \liminf _{q \rightarrow 1} \int_{S} h_{q}\left(e\left(v_{q}\right)\right) d \hat{x} \geq \liminf _{q \rightarrow 1} \sum_{i \in I_{\eta}} \int_{\tilde{\omega}_{i}} h_{q}\left(\rho_{\delta} *\left(\theta_{\delta} e\left(v_{q}\right)\right)\right) d \hat{x} \\
& \geq \sum_{i \in I_{\eta}} h\left(\int_{\tilde{\omega}_{i}} \rho_{\delta} *\left(\theta_{\delta} e(v)\right)\right) \\
& \geq \sum_{i \in I_{\eta}}\left|\omega_{i}\right| h\left(\frac{1}{\left|\omega_{i}\right|} \int_{\omega_{i}} \rho_{\delta} *\left(\theta_{\delta} e(v)\right)\right) \\
&-L\left|\rho_{\delta} *\left(\theta_{\delta} e(v)\right)\right|\left(S \backslash \bigcup_{i \in I_{\eta}} \tilde{\omega}_{i}\right) .
\end{aligned}
$$

The first term of the second member is a Riemann sum. Since moreover $\rho_{\delta} *$ $\theta_{\delta} e(v)$ is a smooth function, by letting $\eta \rightarrow 0$, we obtain

$$
\liminf _{q \rightarrow 1} \int_{S} h_{q}\left(e\left(v_{q}\right)\right) d \hat{x} \geq \int_{S} h\left(\rho_{\delta} * \theta_{\delta} e(v)\right) .
$$

Noticing that $\rho_{\delta} * \theta_{\delta} e(v) \rightarrow e(v)$ in $\mathcal{M}\left(S, \mathbf{M}_{s}^{2 \times 2}\right)$, estimate (26) is obtained by letting $\delta \rightarrow 0$.

Proposition 7 (Upper bound). For every $u \in W_{\Gamma_{0}}^{1, p}\left(\Omega, \mathbf{R}^{3}\right)$ there exists $u_{q}$ weakly converging to $u$ in $W_{\Gamma_{0}}^{1, p}\left(\Omega, \mathbf{R}^{3}\right)$ such that

$$
\limsup _{q \rightarrow 1} \mathcal{F}_{q}\left(u_{q}\right) \leq \mathcal{F}_{0}(u)
$$

or, equivalently, $\Gamma-\lim \sup \mathcal{F}_{q} \leq \mathcal{F}_{0}$.

Proof. Consider the following subset $\mathcal{B}^{1}$ of $\mathcal{B}$ :

$$
\mathcal{B}^{1}:=\left\{u \in W_{\Gamma_{0}}^{1, p}\left(\Omega, \mathbf{R}^{3}\right): \gamma_{S}(u) \in \mathcal{C}^{1}\left(\bar{S}, \mathbf{R}^{3}\right)\right\}
$$


and the functional $\tilde{\mathcal{F}}_{0}: W_{\Gamma_{0}}^{1, p}\left(\Omega, \mathbf{R}^{3}\right) \rightarrow \mathbf{R}^{+}\{+\infty\}$ defined by

$$
\tilde{\mathcal{F}}_{0}(u)=\left\{\begin{array}{l}
\int_{\Omega} f(\nabla u) d x+\int_{S} h\left(e\left(\gamma_{S}(u)\right)\right) d \hat{x} \text { if } u \in \mathcal{B}^{1} \\
+\infty \text { otherwise. }
\end{array}\right.
$$

Take $u \in \mathcal{B}^{1}$ and set $v:=\gamma_{s}(u)$. Let $\left(v_{n}\right)_{n \in \mathbf{N}^{*}}$ be a sequence of continuous piecewise affine functions satisfying $\left\|v_{n}-v\right\|_{W^{1,1}\left(S, \mathbf{R}^{2}\right)} \leq 1 / n$, and consider a sequence $\left(u_{n}\right)_{n \in \mathbf{N}}$ weakly converging to $u$ in $W_{\Gamma_{0}}^{1, p}\left(\Omega, \mathbf{R}^{3}\right)$ satisfying

$$
\begin{aligned}
& \lim _{n \rightarrow+\infty} \int_{\Omega} f\left(\nabla u_{n}\right) d x=\int_{\Omega} f(\nabla u) d x ; \\
& \gamma_{S}\left(\hat{u}_{n}\right)=v_{n} .
\end{aligned}
$$

Such a sequence exists from step 1 of the proof of Proposition 4 in previous section and $u_{n}$ belongs to $\mathcal{B}_{q}$. Writting $e\left(v_{n}\right)=\sum_{i \in I_{n}} a_{i, n} 1_{S_{i, n}}$ where $\left(S_{i, n}\right)_{i \in I_{n}}$ is a finite partition of $S$, and $a_{i, n} \in \mathbf{M}_{s}^{2 \times 2}$, the following estimate holds:

$$
\begin{aligned}
\lim _{q \rightarrow 1}\left(\int_{\Omega} f\left(\nabla u_{n}\right) d x+\int_{S} h_{q}\left(e\left(v_{n}\right)\right) d \hat{x}\right) & =\int_{\Omega} f\left(\nabla u_{n}\right) d x+\lim _{q \rightarrow 1} \sum_{i \in I_{n}} h_{q}\left(a_{i, n}\right)\left|S_{i, n}\right| \\
& =\int_{\Omega} f\left(\nabla u_{n}\right) d x+\sum_{i \in I_{n}} h\left(a_{i, n}\right)\left|S_{i, n}\right| \\
& =\int_{\Omega} f\left(\nabla u_{n}\right) d x+\int_{S} h\left(e\left(v_{n}\right)\right) d \hat{x} . \quad(27)
\end{aligned}
$$

Letting $n \rightarrow+\infty,(27)$ yields

$$
\lim _{n \rightarrow+\infty} \lim _{q \rightarrow 1}\left(\int_{\Omega} f\left(\nabla u_{n}\right) d x+\int_{S} h_{q}\left(e\left(v_{n}\right)\right) d \hat{x}\right)=\int_{\Omega} f(\nabla u) d x+\int_{S} h(e(v)) d \hat{x} .
$$

Then, by using a standard diagonalization argument, there exists a map $q \mapsto$ $n(q)$ such that

$$
\begin{aligned}
& \lim _{q \rightarrow 1} \mathcal{F}_{q}\left(u_{n(q)}\right)=\tilde{\mathcal{F}}_{0}(u) ; \\
& u_{n(q)} \rightarrow u,
\end{aligned}
$$

which implies

$$
\inf \left\{\limsup _{p \rightarrow 1} \mathcal{F}_{q}\left(u_{q}\right): u_{q} \rightarrow u \text { in } W_{\Gamma_{0}}^{1, p}\left(\Omega, \mathbf{R}^{3}\right)\right\} \leq \tilde{\mathcal{F}}_{0}(u)
$$

for all $u \in W_{\Gamma_{0}}^{1, p}\left(\Omega, \mathbf{R}^{3}\right)$. Thus $\Gamma-\lim \sup \mathcal{F}_{q} \leq \tilde{\mathcal{F}}_{0}$. The conclusion of Proposition 7 follows by taking the lower semi-continuous envelop of each two functionals for the weak topology of $W_{\Gamma_{0}}^{1, p}\left(\Omega, \mathbf{R}^{3}\right)$ and by using Lemma 3 .

Corollary 2. Assume that $h_{q}$ satisfies the additional coerciveness condition: there exists $\alpha_{q}>0$ such that $\alpha_{q}|\xi|^{q} \leq h_{q}(\xi)$ for all $\xi \in \mathbf{M}_{s}^{2 \times 2}$. Then

i) The problem $\min \left\{\mathcal{F}_{q}(u)-L(u)\right\}$ possesses at least a solution $\bar{u}_{q}$; 
ii) There exists a subsequence of $\left(\bar{u}_{q}\right)_{q \in(1, p)}$ and $\bar{u}$ solution of the problem $\min \left\{\mathcal{F}_{0}(u)-\langle L, u\rangle\right\}$ such that $\bar{u}_{q} \rightarrow \bar{u}$ in $W_{\Gamma_{0}}^{1, p}\left(\Omega, \mathbf{R}^{3}\right)$.

Proof. The first assertion is obtained by using the direct method in the calculus of variations. The second assertion is a straightforward consequence of variational properties of the $\Gamma$-convergence.

\section{References}

1. E. Acerbi and G. Buttazzo and D. Percivale. Thin inclusions in linear elasticity: a variational approach. J. Reine Angew. Math., 386 (1988), 99-115.

2. O. Anza Hafsa, J. P. Mandallena. Interchange of infimum and integral. Calc. Var. 18 (2003), 433-449.

3. L. Ambrosio, A. Coscia, G. Dal Maso. Fine Properties of Functions in BD. Arch. Rational Mech. Anal. 139, (1997), 201-238.

4. L. Ambrosio, N. Fusco, D. Pallara. Functions of bounded variation and free discontinuity problems. Oxford Mathematical Monographs, Oxford University Press, New York, 2000.

5. H. Attouch. Variational Convergence for Functions and Operators. Applicable Mathematics Series. Pitman Advanced Publishing Program, 1985.

6. H. Attouch, G. Buttazzo, G. Michaille. Variational analysis in Sobolev and BV space: application to PDEs and Optimization. MPS-SIAM Book Series on Optimization 6, December 2005.

7. A. Laure Bessoud, F. Krasucki, G. Michaille. Variational convergence of energy functionals for elastic materials with $\varepsilon$-thin strong inclusions growing as $p(\varepsilon), p(\varepsilon) \rightarrow 1^{+}$. In progress.

8. A. Laure Bessoud, F. Krasucki, G. Michaille. Multi-material with strong interface: Variational modelings. To appear in Asymptotic Analysis.

9. A. C. Barroso, I. Fonseca, R. Toader. A relaxation Theorem in the space of Functions of Bounded Deformation. Ann. Scuola Norm. Sup. Pisa Cl. Sci. 4, Vol XXIX (2000), 19-49.

10. M. Chipot, D. Kinderlehrer. Equilibrium configurations of crystals. Arch. Rat. Mech. Anal. 103 (1988), 237-277.

11. G. Dal Maso. An introduction to $\Gamma$-convergence. Birkäuser, Boston, 1993.

12. F. Demengel, R. Temam. Convex functions of measures and applications. Indiana Univ. Math. J. 33 (1984), 673-709.

13. R.V. Kohn. The relaxation of a double-well energy. Continuum Mech. Thermodyn. 3 (1991), 193-236.

14. H. Le Dret, A. Raoult. The nonlinear membrane model as Variational limit in nonlinear three-dimensional elasticity. J. Math. Pures Appl., IX. Ser. 74 (1995), no 6, 549-578. 
15. C. Licht-G. Michaille. A modelling of elastic adhesive bonding joints. Advances in Mathematical Sciences and Applications, 7, no. 2 (1997), 711-740.

16. C. Licht-G. Michaille. A nonlocal energy functional in pseudo-plasticity. Asymptotic Analysis, 45, (2005), 313-339.

17. R.Temam. Problèmes Mathématiques en Plasticité. Gauthier-Villars, Paris 1983. 